\title{
Dufour and Soret Effects on MHD Forced Convective Heat and Mass Transfer Flow of Non-Newtonian Power Law Fluid with Thermal Radiation and Viscous Dissipation
}

\author{
Chinmayee Podder ${ }^{1, *}$, Md. Abdus Samad ${ }^{2}$ \\ ${ }^{1}$ Department of Mathematics, University of Barisal, Barisal, Bangladesh \\ ${ }^{2}$ Department of Applied Mathematics, University of Dhaka, Dhaka, Bangladesh
}

Email address:

chinmayeepodder1@gmail.com (C. Podder)

${ }^{*}$ Corresponding author

\section{To cite this article:}

Chinmayee Podder, Md. Abdus Samad. Dufour and Soret Effects on MHD Forced Convective Heat and Mass Transfer Flow of NonNewtonian Power Law Fluid with Thermal Radiation and Viscous Dissipation. American Journal of Applied Mathematics. Vol. 4, No. 6, 2016, pp. 296-309. doi: 10.11648/j.ajam.20160406.16

Received: September 26, 2016; Accepted: November 1, 2016; Published: November 29, 2016

\begin{abstract}
This paper investigates the Dufour and Soret effects of forced convection heat and mass transfer of an electrically conducting, non-Newtonian power-law fluid past a stretching sheet under the simultaneous action of suction, radiation, uniform transverse magnetic field, heat generation and viscous dissipation. The stretching sheet is assumed to continuously moving with a power-law velocity and maintaining a uniform surface heat flux. The governing nonlinear partial differential equations are transformed into a system of non linear ordinary differential equations using appropriate similarity transformations. The resulting dimensionless equations are solved numerically using sixth order Runge-Kutta integration scheme with Nachtsheim-Swigert shooting iterative technique. A systematical study of numerical results for the nondimensional velocity, temperature and concentration profiles are presented graphically. The viscous drag or local Skin-friction coefficient, heat transfer rate or local Nusselt number and mass transfer rate or local Sherwood number are represented in tabular and graphical forms to illustrate the details of flow characteristics and their dependence on all physically important parameters in case of Newtonian and non-Newtonian (pseudo-plastic and dilatants) fluids.
\end{abstract}

Keywords: Dufour Number, Soret Number, Non-Newtonian Power-Law Fluid, Thermal Radiation, Viscous Dissipation

\section{Introduction}

The heat, mass and momentum transfer in the laminar boundary layer flow of non-Newtonian power law fluid on stretching sheets are important from a theoretical as well as practical point of view because of their wider applications to polymer technology, metallurgy, many mechanical forming processes, such as extrusion, melt-spinning, cooling, manufacture of plastic and rubber sheets, glass blowing, continuous casting and spinning of fibers etc. The interaction of radiation with hydromagnetic flow has become industrially more prominent in the processes wherever high temperatures occur. Nuclear power plants, gas turbines and the various propulsion devices for aircrafts, missiles, satellites and space vehicles are examples of such engineering areas. Forced convection should be considered as one of the main methods of useful heat transfer as significant amounts of heat energy can be transported very efficiently and this mechanism is found very commonly in everyday life, including central heating, air conditioning, steam turbines and designing or analyzing heat exchangers, pipe flow, and flow over a plate at a different temperature than the stream. Dufour effect is the inverse phenomenon of thermal diffusion. If two chemically different nonreacting gases or liquids, which were initially at the same temperature, are allowed to diffuse into each other, then there arises a difference of temperatures in the system. The difference in temperatures is retained if a concentration gradient is maintained. Soret effect (thermodiffusion) is the diffusion of material in an unevenly heated mixture of gases or a solution caused by the presence of a temperature gradient in the system. This normally applies to liquid mixtures, which behave 
according to different, less well-understood mechanisms than gaseous mixtures. It has been utilized for isotope separation and in mixture between gases with very light molecular weight and of medium molecular weight. Both Soret and Dufour effects are significant when density differences exist in the flow regime.

Some literature surveys and reviews of pertinent work in this field are documented by Elbashbeshy [1] investigated heat transfer over a stretching surface with variable and uniform surface heat flux subject to injection and suction. Vajravelu and Hadjinicolaou [2] studied the convective heat transfer in an electrically conducting fluid near an isothermal stretching sheet. Howell et al. [3] analyzed momentum and heat transfer on a continuous moving surface in a power law fluid. Rahman et al. [4] investigated MHD forced convective flow of a micropolar fluid past a non-linear stretching sheet with a variable viscosity. The MHD boundary layer flow over a continuously moving plate for a micropolar fluid has been studied by Raptis [5]. Anderson et al. [6] and Mahmoud and Mahmoud [7] adopted the non-linearity relation as power-law dependency of shear stress on rate of strain. Dandapaat et al. [8] extended the problem to study heat transfer and Datti et al. [9] analyzed the problem over a non-isothermal stretching sheet. Cess [10] studied to determine the influence of radiative heat transfer upon the forced convection. Pop et al. [11] studied radiation effect on the flow near the stagnation point of a stretching sheet. Damesh et al. [12] investigated the MHD forced convection heat transfer from radiate surfaces in the presence of a uniform transverse magnetic field with conductive fluid suction or injection from a porous plate. Cortell [13] analyzed the effects of viscous dissipation and radiation on the thermal boundary layer over a nonlinearly stretching sheet. Chen [14] studied the effects of magnetic field and suction/injection on the flow of power-law non-Newtonian fluid over a power law stretched sheet subject to a surface heat flux. O. D. Makinde et al. [15] studied chemically-reacting hydromagnetic boundary layer flow with Soret/Dufour effects and a convective surface boundary condition. M. J. Subhakar et al. [16] analyzed Soret and Dufour effects on MHD convective flow of heat and mass transfer over a moving non-isothermal vertical plate with heat generation/absorption. M. S. Alam et al. [17] investigated Dufour and Soret effects on unsteady MHD free convection and mass transfer flow past a vertical porous plate in a porous medium. Mahdy [18] studied Soret and Dufour effect on double diffusion mixed convection from a vertical surface in a porous medium saturated with a non-Newtonian fluid. Chen et al. [19] analyzed Soret and Dufour effects on free convection flow of non-Newtonian fluids along a vertical plate embedded in a porous medium with thermal radiation. Abreu et. al. [20] discussed about boundary layer flows with Dufour and Soret effects on forced and natural convection. In this study, the problem studied by Chen [14] has been extended to investigate the effects of thermal radiation, heat generation and viscous dissipation.

Numerical and graphical computations for the velocity, temperature and concentration profiles have been carried out of different values of Suction parameter $\left(f_{w}\right)$, Prandtl number(Pr), Magnetic parameter $(M)$, Radiation parameter $(N)$, Heat source parameter $(Q)$, Schmidt number $(S c)$, Eckert number $(E c)$, velocity index $(p)$, power-law fluid index $(n), \operatorname{Dufour}(D u)$ and Soret $(S r)$ numbers. The local skin friction coefficient, local Nusselt number and local Sherwood number have also been obtained to investigate more practical and physical effect of parameters on Newtonian and non-Newtonian fluids.

\section{Numerical Formulation}

Let us consider a steady two dimensional MHD forced convective laminar boundary layer flow of a viscous incompressible and electrically conducting fluid obeying the power-law model along a permeable stretching sheet with the influence of thermal radiation, heat generation, viscous dissipation, Soret and Dufour effects. The origin is located at the slit through which the sheet is drowning through the fluid medium. The flow is assumed to be in the $x$-direction, which is taken along the sheet and $y$-axis is normal to it. Two equal and opposite forces are introduced along the $x$-axis, so that the sheet is stretched keeping the origin fixed. The plate is maintained at a constant temperature $T_{w}$ and the ambient temperature is $T_{\infty}$. This continuous sheet is assumed to move with a velocity according to a power-law form, i.e. $u_{w}=C x^{p}$. The fluid is considered to be gray, absorbing emitting radiation but non-scattering medium and the Rosseland approximation is used to describe the radiative heat flux in the energy equation. The radiative heat flux in the $x$-direction is considered negligible in comparison to the $y$-direction. A strong magnetic field $B$ is applied in $y$-direction. The magnetic Reynolds number is assumed to be small so that induced magnetic field is negligible. The electrical current flowing in the fluid gives rise to an induced magnetic field if the fluid were an electrical insulator, but here have taken the fluid to be the electrically conducting. Hence, only the applied magnetic field $B$ plays a role which gives rise to magnetic forces $F_{x}=$ $\frac{\sigma B u}{\rho}$ in the $x$-direction. Under the usual boundary layer approximation, the flow, heat and mass transfer in the presence of thermal radiation, heat generation, viscous dissipation and Dufour/Soret effects are governed by the following equations: Continuity Equation:

$$
\frac{\partial u}{\partial x}+\frac{\partial v}{\partial y}=0
$$

Momentum Equation:

$$
u \frac{\partial u}{\partial x}+v \frac{\partial u}{\partial y}=\frac{K}{\rho} \frac{\partial}{\partial y}\left(\left|\frac{\partial u}{\partial y}\right|^{n-1} \frac{\partial u}{\partial y}\right)-\frac{\sigma B^{2} u}{\rho}
$$

Energy Equation:

$$
\begin{aligned}
& u \frac{\partial T}{\partial x}+v \frac{\partial T}{\partial y}=\alpha \frac{\partial^{2} T}{\partial y^{2}}+\frac{Q_{0}}{\rho C_{p}}\left(T-T_{\infty}\right)-\frac{1}{\rho C_{p}} \frac{\partial q_{r}}{\partial y}+ \\
& \frac{K}{\rho C_{p}}\left(\left|\frac{\partial u}{\partial y}\right|^{n-1} \frac{\partial u}{\partial y}\right)^{2}+\frac{D_{m} K_{T}}{\rho C_{p} C_{s}} \frac{\partial^{2} C}{\partial y^{2}}
\end{aligned}
$$

Concentration Equation:

$$
u \frac{\partial C}{\partial x}+v \frac{\partial C}{\partial y}=D_{m} \frac{\partial^{2} C}{\partial y^{2}}+\frac{D_{m} K_{T}}{T_{m}} \frac{\partial^{2} T}{\partial y^{2}}
$$

where $u$ and $v$ are the velocity components along $x$ and $y$ directions respectively, $T$ be the temperature of the fluid 
layer, $v$ is the kinematic viscosity, $\rho$ is the density, $\sigma$ is the electric conductivity, $\kappa$ is the thermal conductivity, $B$ is the uniform magnetic field, $C_{p}$ is the specific heat at constant pressure, $\alpha$ is the thermal diffusivity, $q_{r}$ is the radiative heat flux, $K$ is the consistency coefficient, $n$ is the flow behavior/power-law fluid index, $D_{m}$ is mass diffusivity, $K_{T}$ is thermal diffusion ratio, $C_{s}$ is concentration susceptibility and $T_{m}$ is mean fluid temperature. The radiative heat flux $q_{r}$ is described by the Rosseland approximation such that,

$$
q_{r}=-\frac{4 \sigma_{1}}{3 k_{1}} \frac{\partial T^{4}}{\partial y}
$$

where $\sigma_{1}$ is the Stefan-Boltzman constant and $k_{1}$ is the Rosselend mean absorption coefficient. It is assumed that the temperature difference within the flow are sufficiently small such that $T^{4}$ in a Taylor series about the free steam temperature $T_{\infty}$ and then neglecting higher-order terms. This results in the following approximation:

$$
T^{4} \approx 4 T_{\infty}^{3}-3 T_{\infty}^{4}
$$

Using (5) and (6) in $\frac{\partial q_{r}}{\partial y}$ which appear in equation (4), we have

$$
\frac{\partial q_{r}}{\partial y}=-\frac{16 \sigma_{1} T_{\infty}^{3}}{3 \kappa_{1}} \frac{\partial^{2} T}{\partial y^{2}}
$$

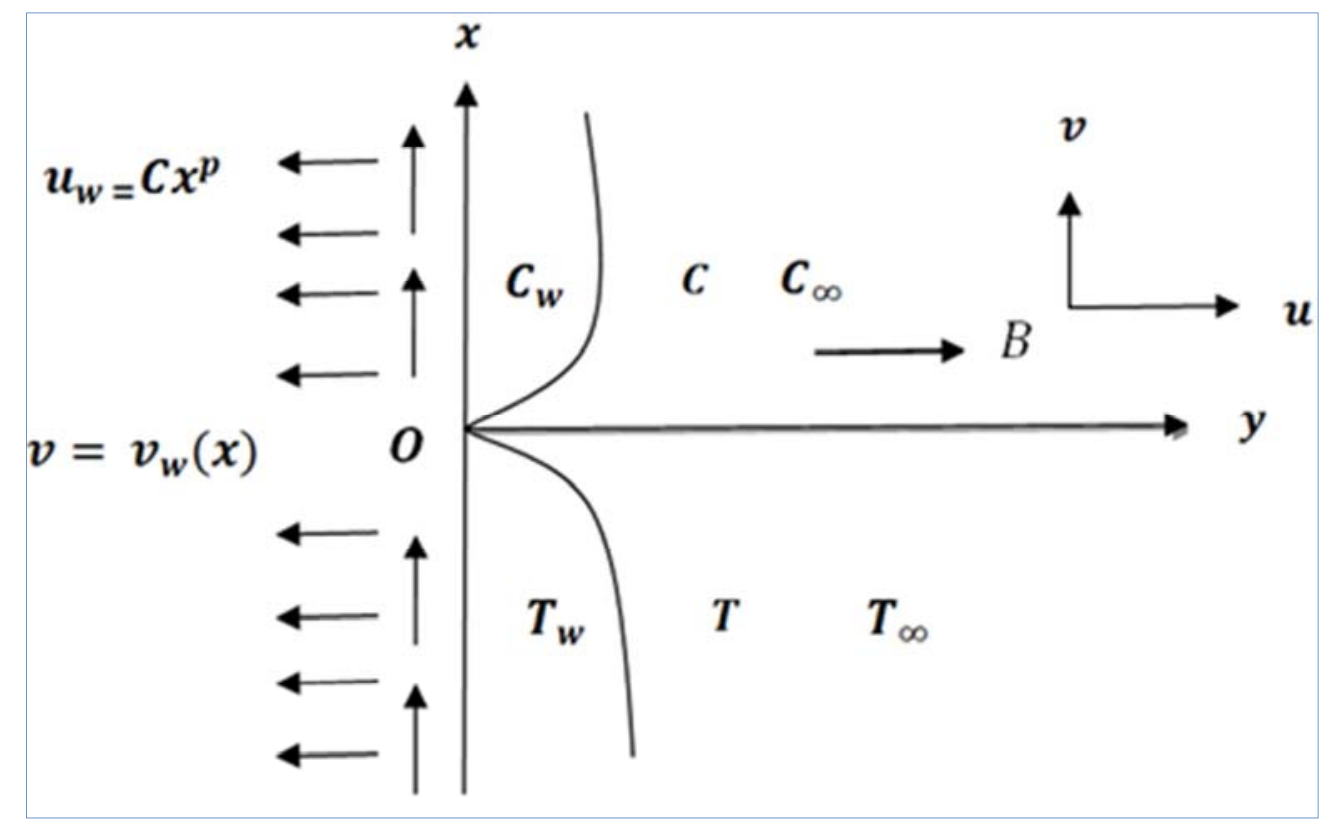

Fig. 1. The physical model of boundary layer.

In order to obtain a similarity solution of the problem, introduce a similarity parameter $\delta(x)$, such that $\delta(x)$ is a length scale. Introducing the following dimensionless quantities, we have

$$
\begin{gathered}
\eta=\frac{y}{\delta(x)}=\left(\frac{C^{2-n}}{K / \rho}\right)^{1 /(n+1)} x^{\{p(2-n)-1\} /(n+1)} y \\
\psi=\left(\frac{C^{1-2 n}}{K / \rho}\right)^{-1 /(n+1)} x^{\frac{\{p(2 n-1)+1\}}{n+1}} f(\eta)
\end{gathered}
$$

Introducing $\frac{\partial q_{r}}{\partial y}$ in (3), the following governing boundary layer equation:

Energy Equation:

$$
\begin{gathered}
u \frac{\partial T}{\partial x}+v \frac{\partial T}{\partial y}=\alpha \frac{\partial^{2} T}{\partial y^{2}}+\frac{Q_{0}}{\rho C_{p}}\left(\mathrm{~T}-\mathrm{T}_{\infty}\right)+\frac{1}{\rho C_{p}} \frac{16 \sigma_{1} T_{\infty}^{3}}{3 k_{1}} \frac{\partial^{2} T}{\partial y^{2}}+ \\
\frac{K}{\rho C_{p}}\left(\left|\frac{\partial u}{\partial y}\right|^{n-1} \frac{\partial u}{\partial y}\right)^{2}+\frac{D_{m} K_{T}}{\rho C_{p} C_{s}} \frac{\partial^{2} C}{\partial y^{2}}
\end{gathered}
$$

The appropriate boundary conditions are:

$$
\left.\begin{array}{cc}
u_{w}=C x^{p}, v=v_{w}, \frac{\partial T}{\partial y}=-\frac{q_{w}}{\kappa}, C=C_{\infty}+b x \text { at } & y=0, x>0 \\
u_{w} \rightarrow 0, T \rightarrow T_{\infty}, C \rightarrow C_{\infty} \text { as } & y \rightarrow \infty
\end{array}\right\}
$$

where $v_{w}$ is the surface mass flux and $q_{w}$ is the surface heat flux. It should be noted that positive $p$ indicates that the surface is accelerated while negative $p$ implies that surface is decelerated from the slit. Positive $v_{w}$ is for fluid injection and negative $v_{w}$ for fluid suction at the sheet surface. The last term in the energy equation (3) has been introduced to investigate the Dufour effect and last term in the concentration equation (4) for Soret effect respectively. 


$$
\begin{aligned}
& u=\frac{\partial \psi}{\partial y}=u_{w} f^{\prime}(\eta) \\
& v=-\frac{\partial \psi}{\partial x}=-u_{w} R e_{x}^{-\frac{1}{(n+1)}}\left[\frac{\{p(2 n-1)+1\}}{(n+1)} f(\eta)+\frac{\{p(2-n)-1\} \eta}{(n+1)} f^{\prime}(\eta)\right]
\end{aligned}
$$

Introducing similarity variables from equations (10)(13) and using the transformations the following equations are found:

Momentum Equation:

$$
\left(\left|f^{\prime \prime}\right|^{n-1} f^{\prime \prime}\right)^{\prime}+\frac{\{p(2 n-1)+1\}}{(n+1)} f f^{\prime \prime}-p\left(f^{\prime}\right)^{2}-M f^{\prime}=0
$$

Energy Equation:

$$
\begin{gathered}
\frac{3 N+4}{3 N \mathrm{Pr}} \theta^{\prime \prime}+\frac{\{p(2-n)-1\}}{(n+1)} f^{\prime} \theta+\frac{\{p(2 n-1)-1\}}{(n+1)} f \theta^{\prime}+Q \theta+ \\
E c\left(\left|f^{\prime \prime}\right|^{n-1} f^{\prime \prime}\right)^{2}+D u \phi^{\prime \prime}=0
\end{gathered}
$$

Concentration Equation:

$$
\frac{1}{S c} \phi^{\prime \prime}+\operatorname{Sr} \theta^{\prime \prime}+\frac{\{p(2 n-1)+1\}}{(n+1)} f \phi^{\prime}-f^{\prime} \phi=0
$$

The transformed boundary conditions are:

$$
\left.\begin{array}{c}
f^{\prime}=1, f=\frac{n+1}{p(2 n-1)+1} f_{w}, \theta^{\prime}=-1, \phi=1 a t \eta=0 \\
f^{\prime} \rightarrow 0, \theta \rightarrow 0, \phi \rightarrow 0 a s \eta \rightarrow \infty
\end{array}\right\}
$$

where $f_{w}=-\frac{v_{w}}{u_{w}} R e_{x}^{\frac{1}{(n+1)}}$ is the suction parameter, $M=$ $\frac{\sigma B^{2} x}{\rho u_{w}}$ is the magnetic field parameter, $R e_{x}=\frac{\rho u_{w}^{2-n} x^{n}}{K}$ is the local Reynolds number, $\operatorname{Pr}=\frac{x u_{w}}{\alpha} R e_{x}^{\frac{-2}{(n+1)}}$ is the generalized Prandtl number, $N=\frac{\kappa \kappa_{1}}{4 \sigma_{1} T_{\infty}^{3}}$ is the radiation parameter, $Q=\frac{Q_{0} x}{u_{w} \rho C_{p}}$ is the heat source parameter, $E c=\frac{\left(u_{w} \frac{n+1}{2}\right)^{2}}{c_{p} \cdot \frac{q_{w}}{k} x^{n}} R e_{x}^{\frac{n}{n+1}} \quad$ is the Eckert number, $D u=$ $\frac{D_{m} K_{T} b k}{\rho C_{p} C_{s} q_{w}}\left(\frac{u_{w}^{3(1-n)} R e_{x}}{(k x / \rho)^{2}}\right)^{\frac{1}{n+1}}$ is the Dufour number, $S c=$ $\frac{u_{w} x^{2}}{D_{m}} R e_{x}^{\frac{-2}{(n+1)}}$ is the local Schmidt number, $S r=$ $\frac{D_{m} K_{T}}{b T_{m}} \frac{q_{W}}{\kappa} \frac{R e_{x}^{\frac{1}{(n+1)}}}{x u_{W}}$ is the Soret number. It is noted that the magnetic field strength $B$ should be proportional to $x$ to the power of $(p-1) / 2$ to eliminate the dependence of $M$ on $x$, i.e. $B(x)=B_{0} x^{(p-1) / 2}$, where $B_{0}$ is a constant.

\section{Results and Discussion}

In order to analyze the results, graphical computations have been carried out for the different values of Suction parameter $\left(f_{w}\right)$, Prandtl number $(\operatorname{Pr})$, Magnetic parameter $(M)$, Radiation parameter $(N)$, Heat source parameter $(Q)$, Schmidt number( $S c)$, Eckert number ( $E c)$, velocity $\operatorname{index}(p)$, power-law fluid index $(n)$, Dufour number $(D u)$ and Soret number(Sr).
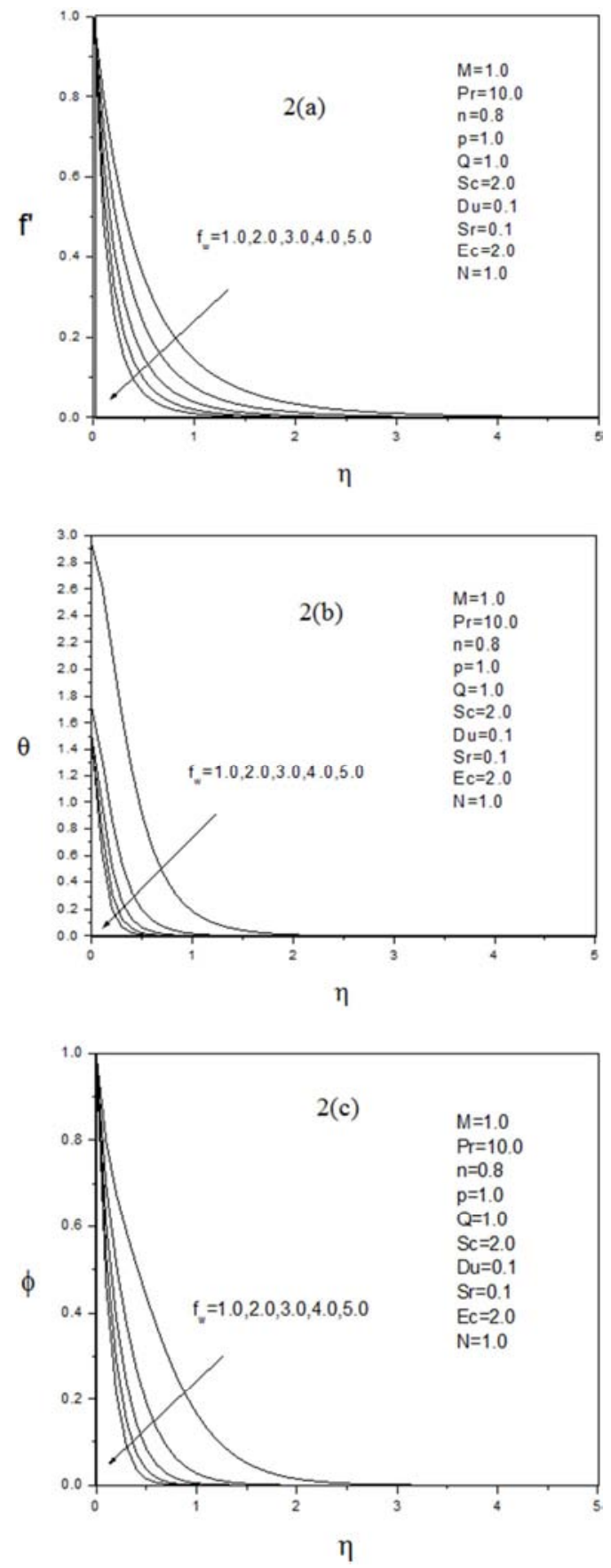

Fig. 2. (a), (b), (c). Suction parameter $\left(f_{w}\right)$ effect on velocity, temperature and concentration profiles.

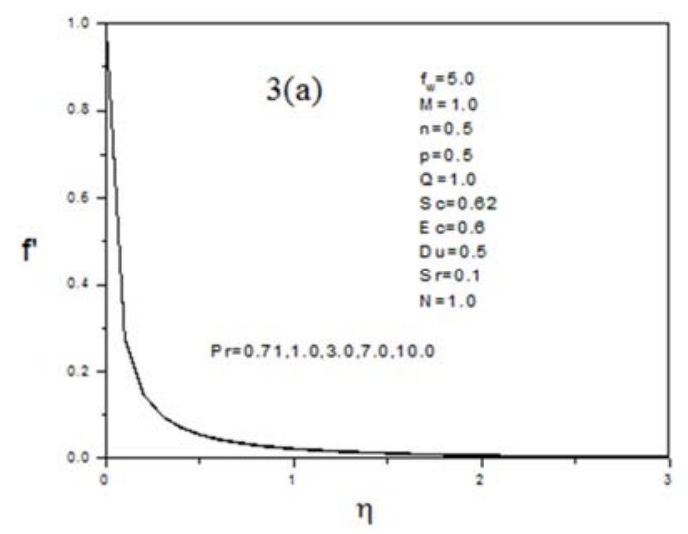



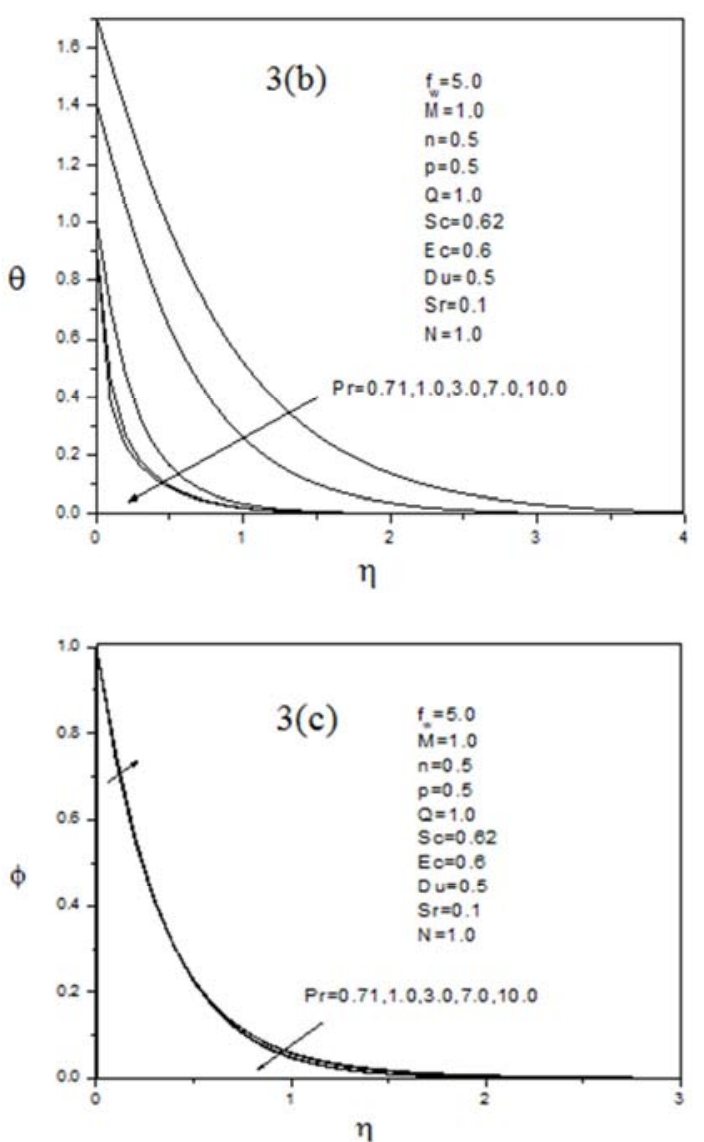

Fig. 3. (a), (b), (c). Prandtl number(Pr) effect on velocity, temperature and concentration profiles
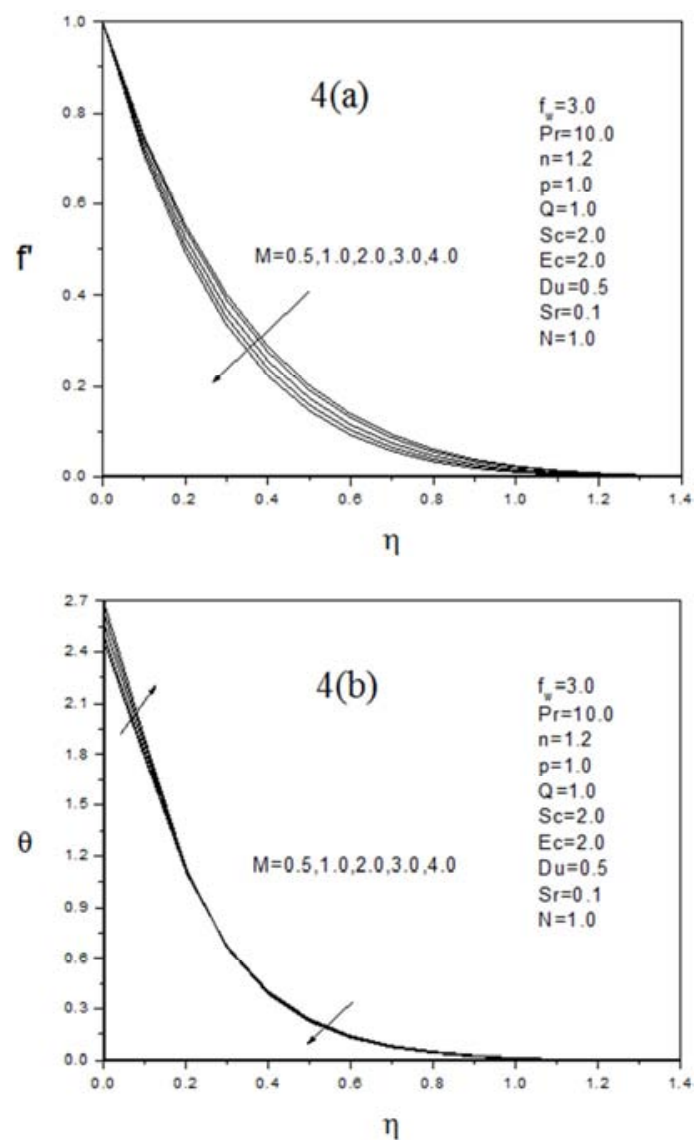

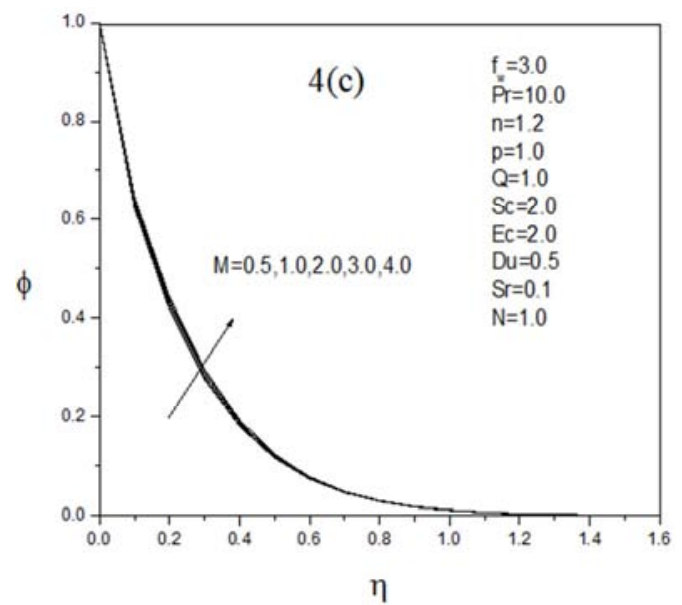

Fig. 4. (a), (b), (c). Magnetic parameter(M) effect on velocity, temperature and concentration profiles.
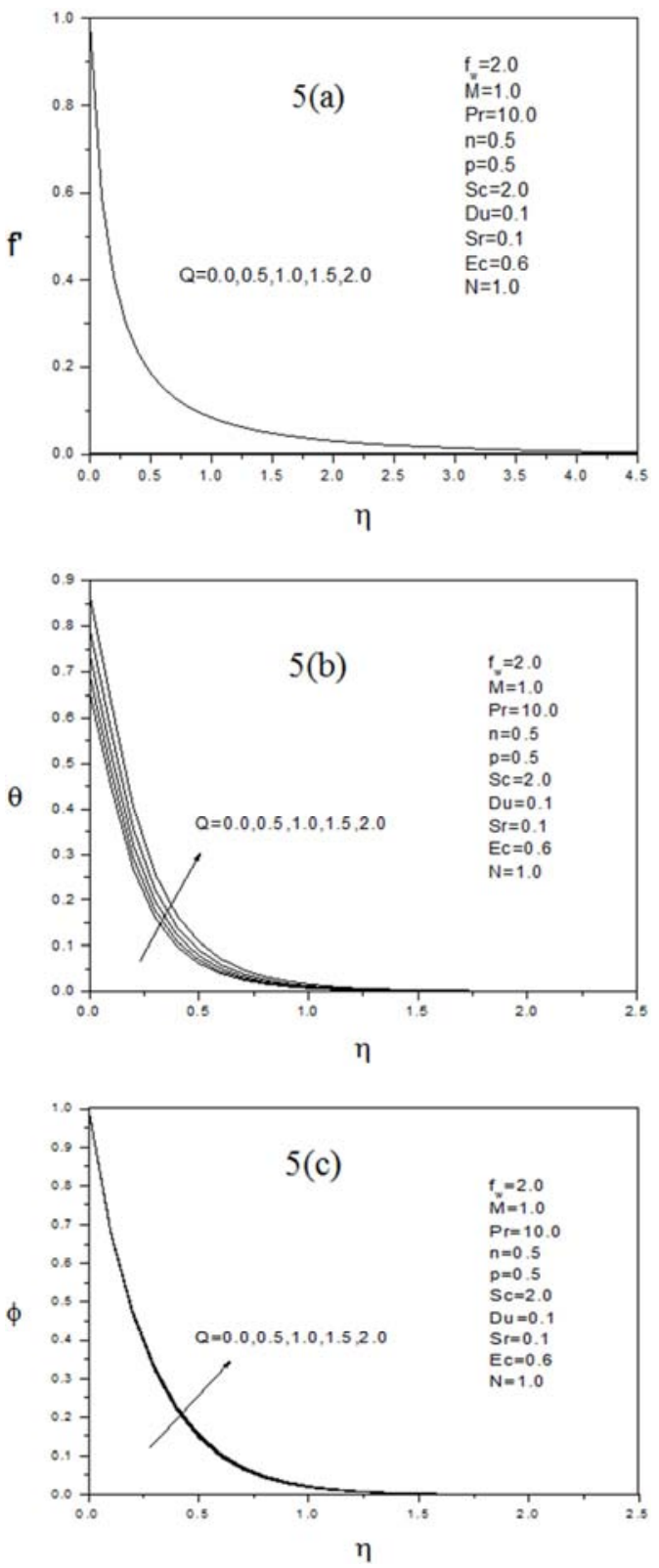

Fig. 5. (a), (b), (c). Heat source parameter effect on velocity, temperature and concentration profiles. 


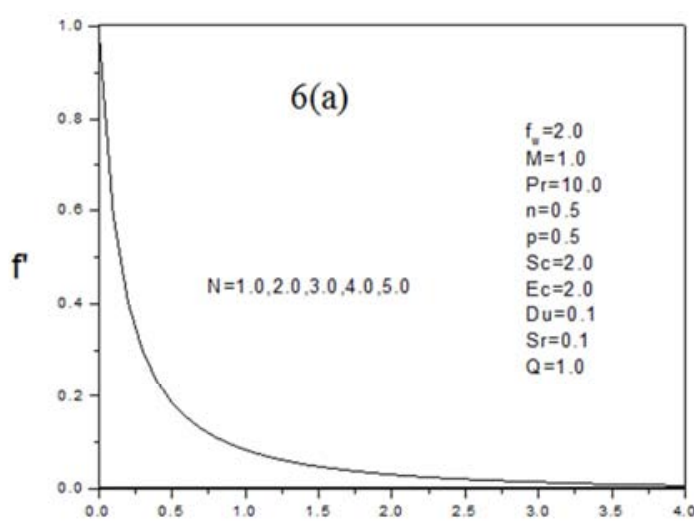

$\eta$
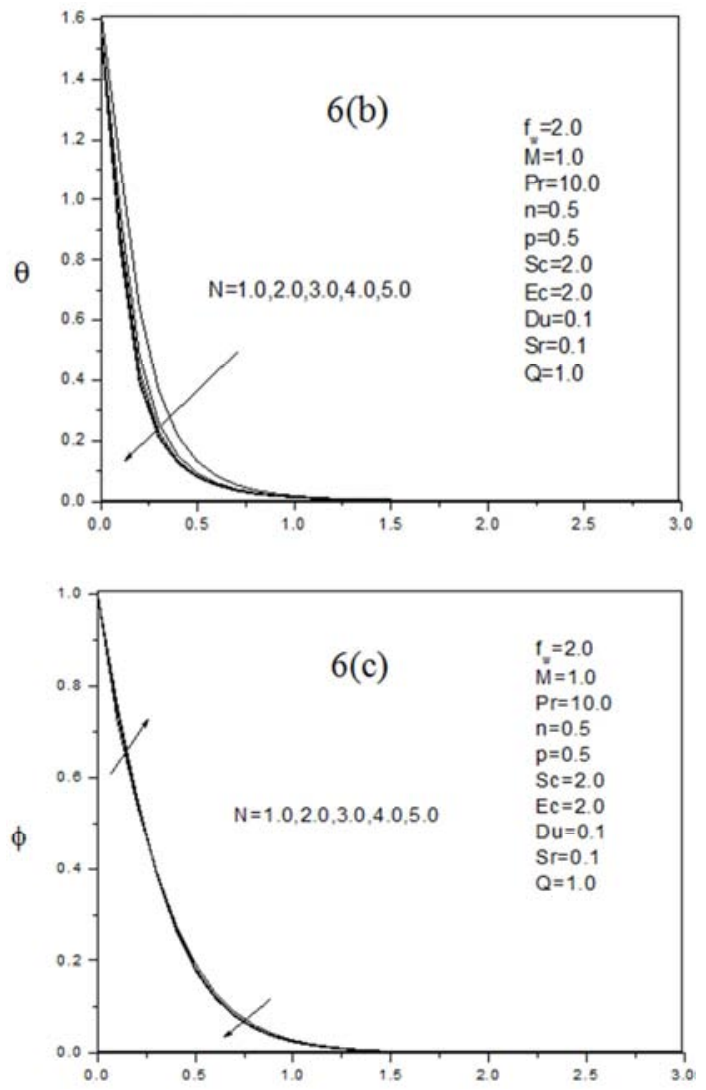

Fig. 6. (a), (b), (c). Radiation parameter (N) effect on velocity, temperature and concentration profiles.

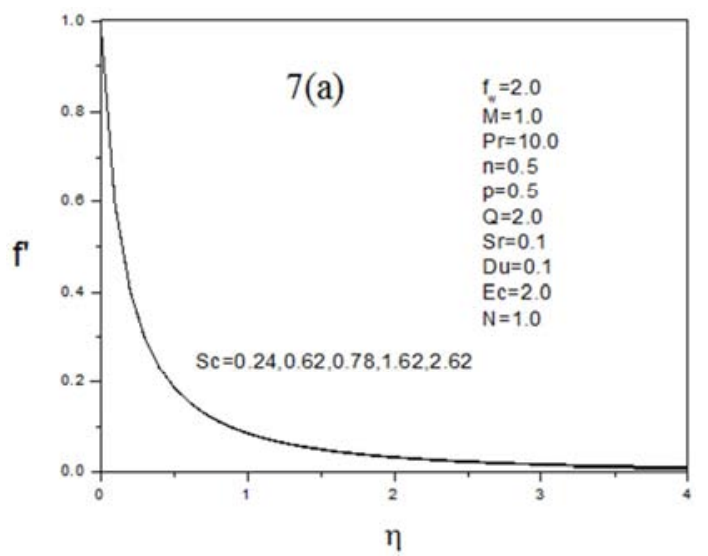

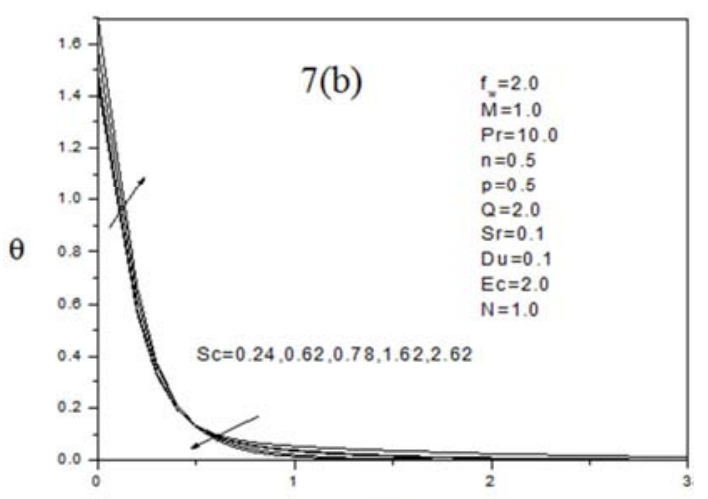

$\eta$

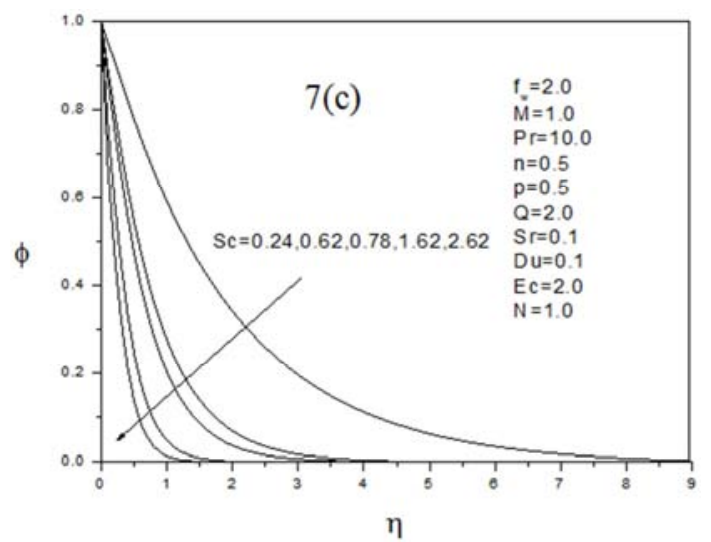

Fig. 7. (a), (b), (c). Schmidt number(Sc) effect on velocity, temperature and concentration profiles.
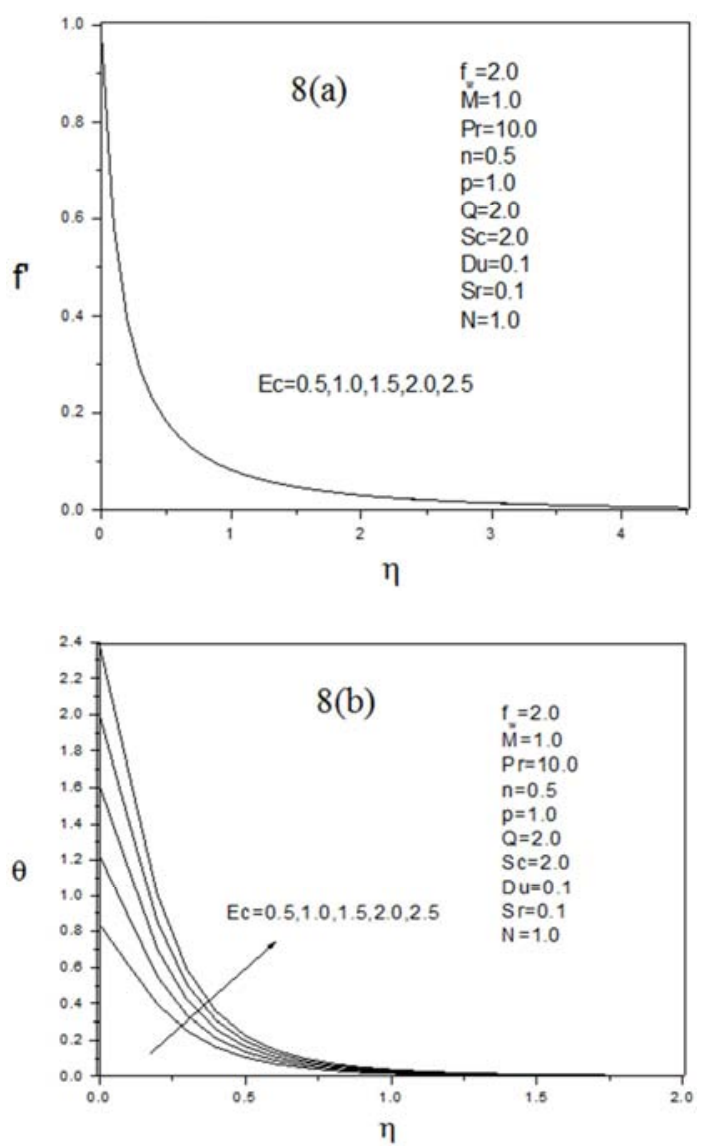


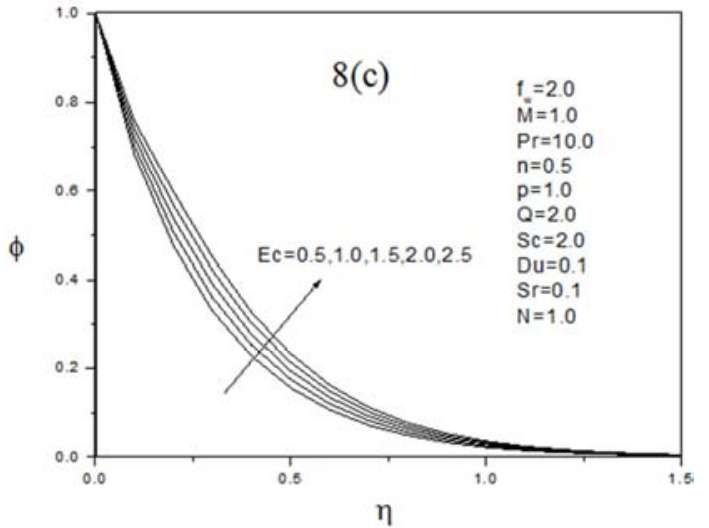

Fig. 8. (a), (b), (c). Eckert number(Ec) effect on velocity, temperature and concentration profiles.
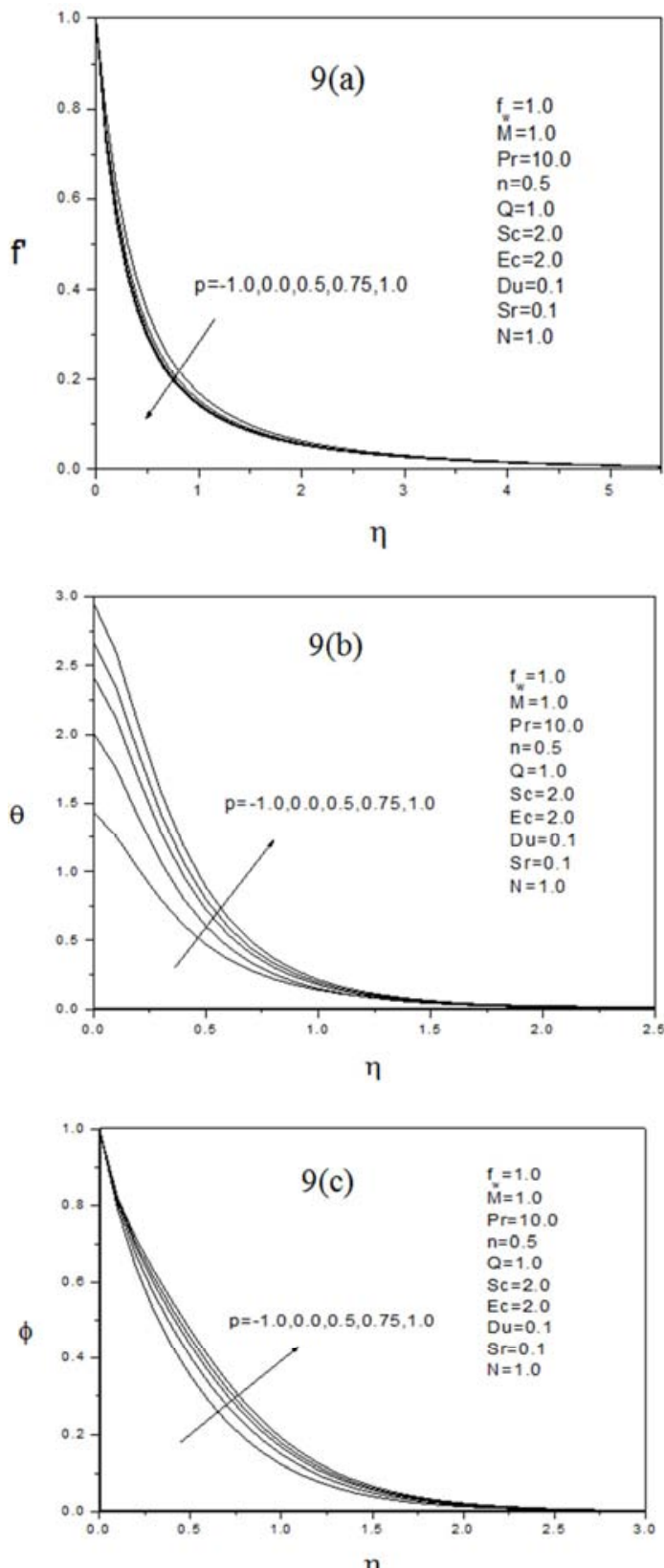

Fig. 9. (a), (b), (c). Velocity index(p)effect on velocity, temperature and concentration profiles for pseudo-plastic fluid $(n=0.5)$.
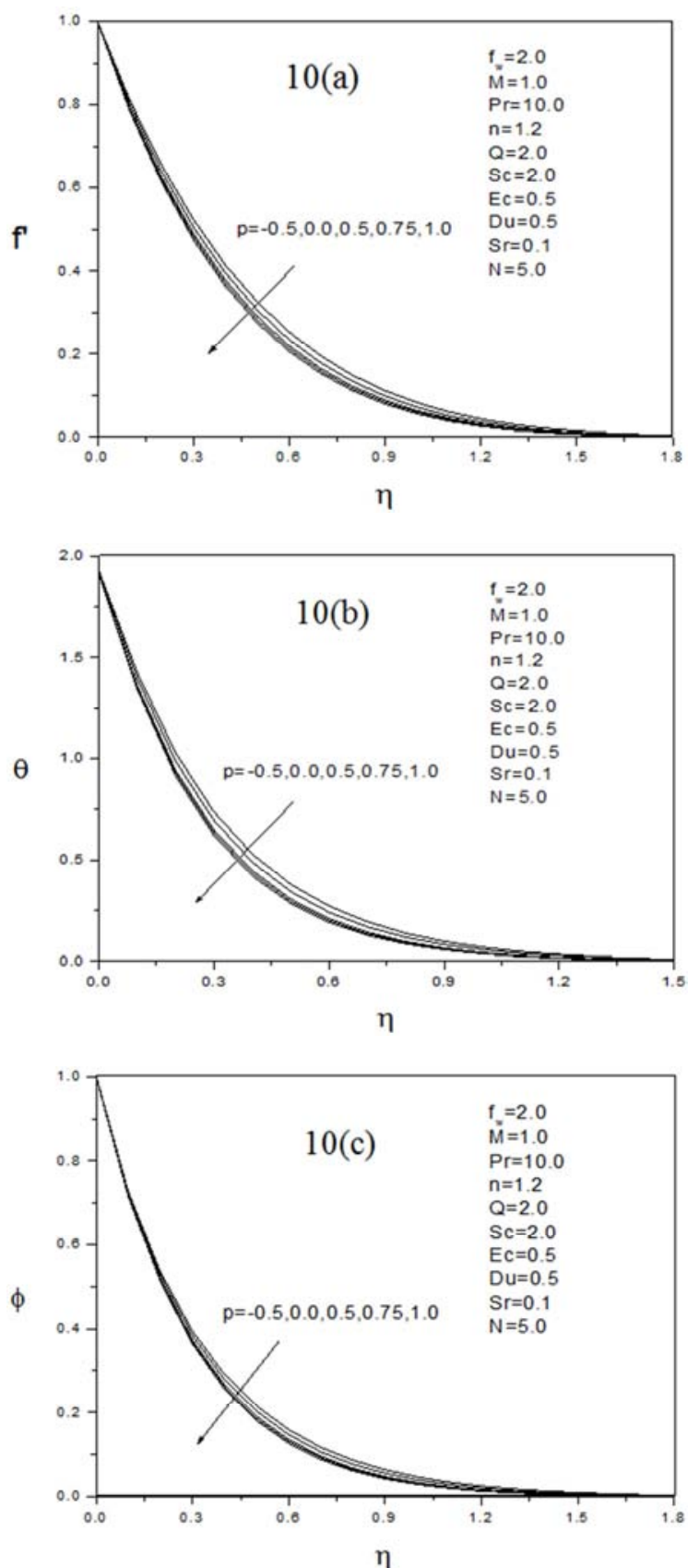

Fig. 10. (a),(b),(c). Velocity index( $p$ )effect on velocity, temperature and concentration profiles for dilatants fluid $(n=1.2)$.

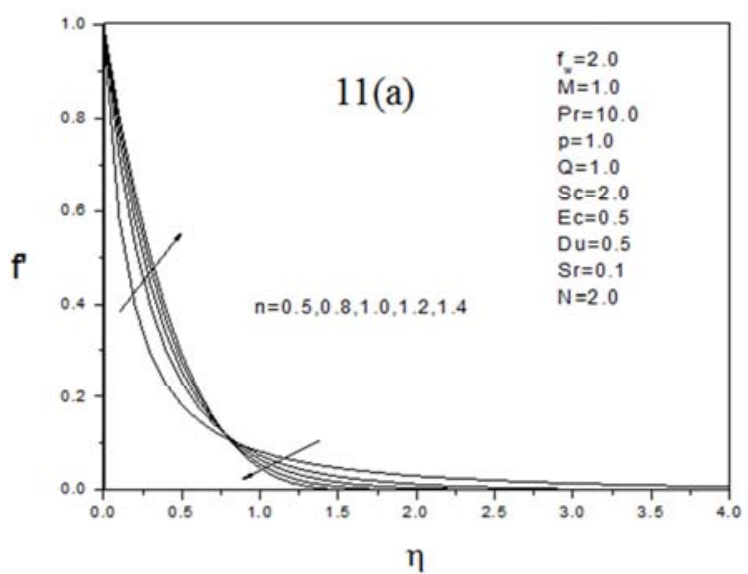



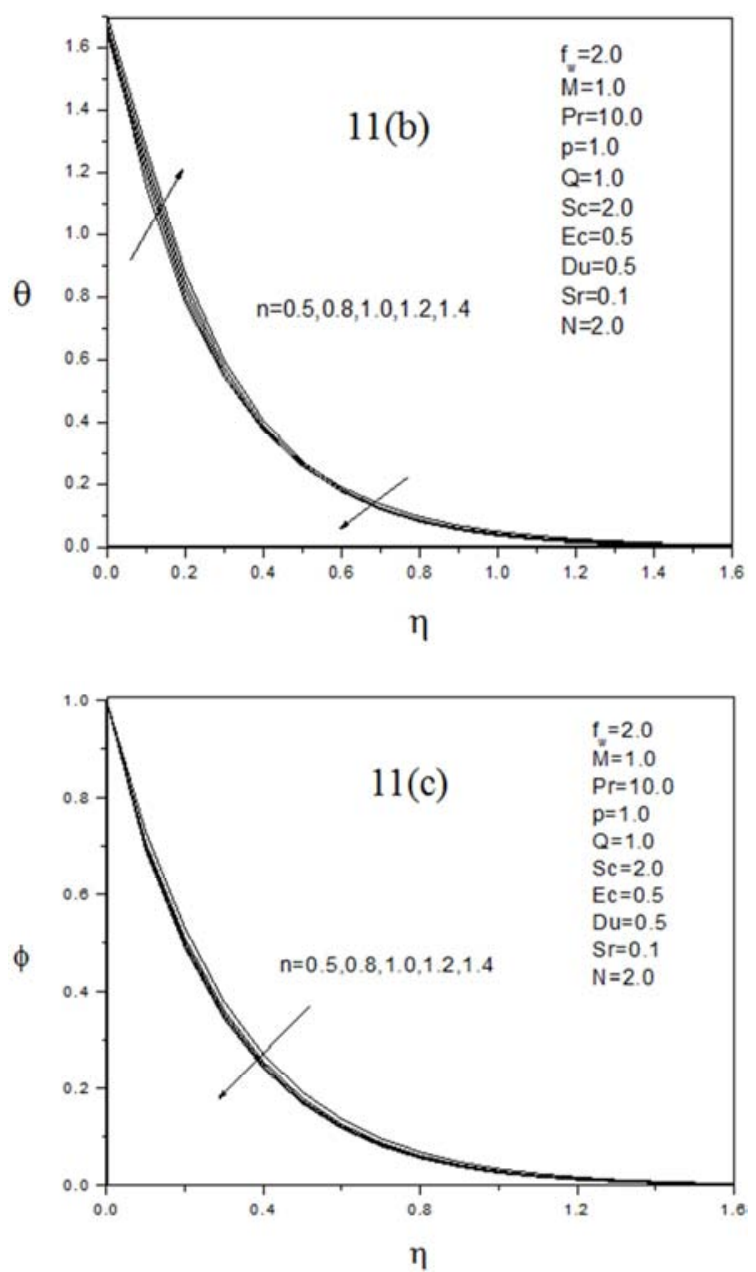

Fig. 11. (a), (b), (c). Power-law index(n) effect on velocity, temperature and concentration profiles for accelerated flow $(p=1.0)$.
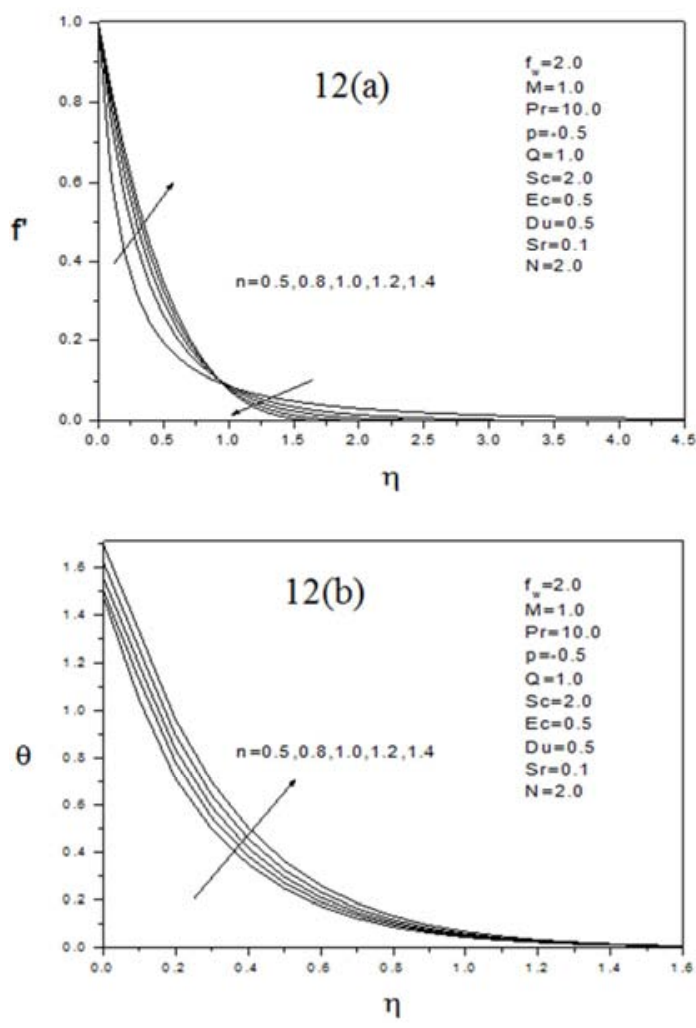

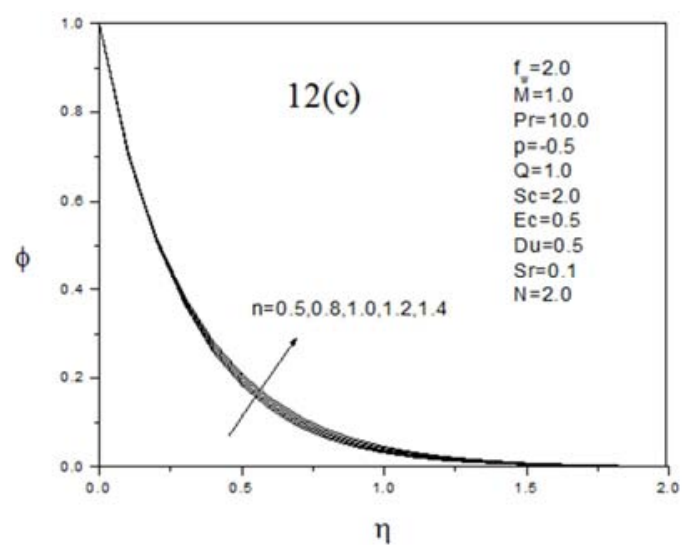

Fig. 12. (a), (b), (c). Power-law index(n) effect on velocity, temperature and concentration profiles for decelerated flow $(p=-0.5)$.
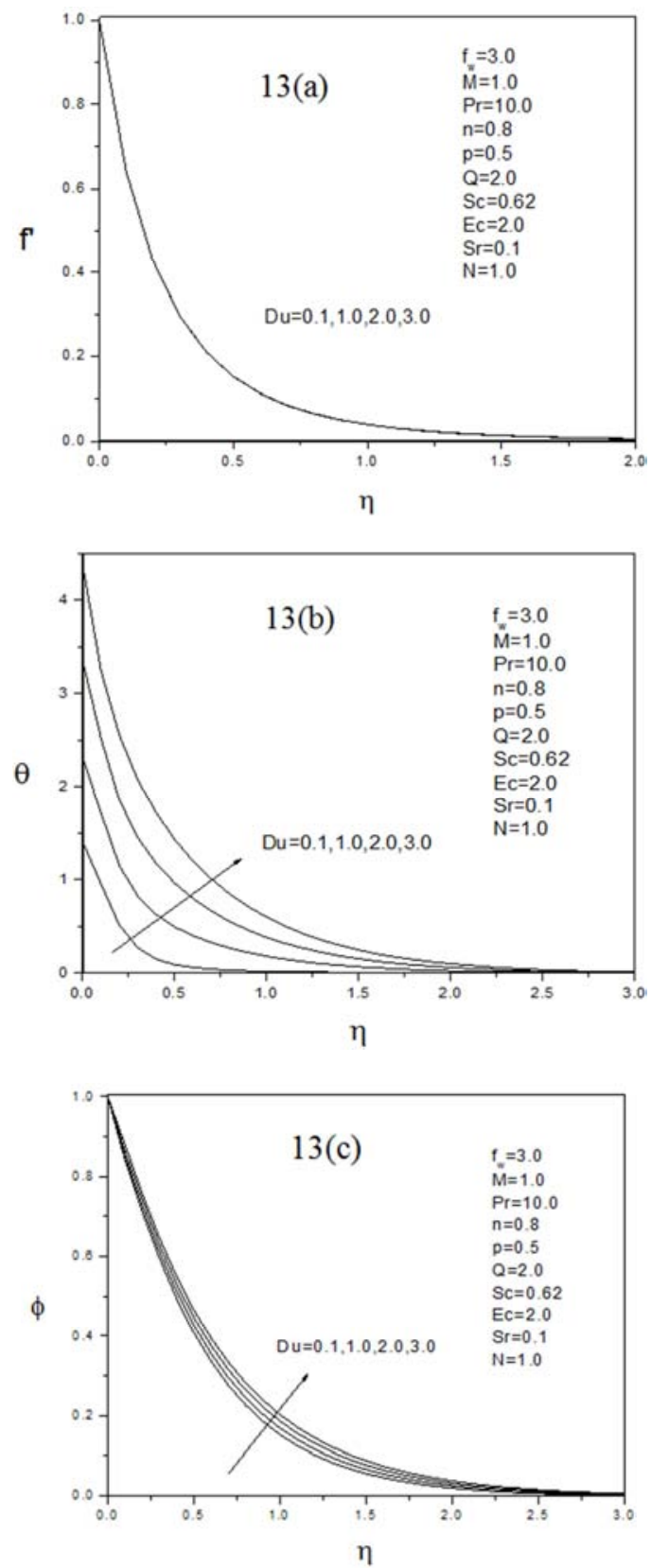

Fig. 13. (a), (b), (c). Dufour effect(Du) on velocity, temperature and concentration profiles. 

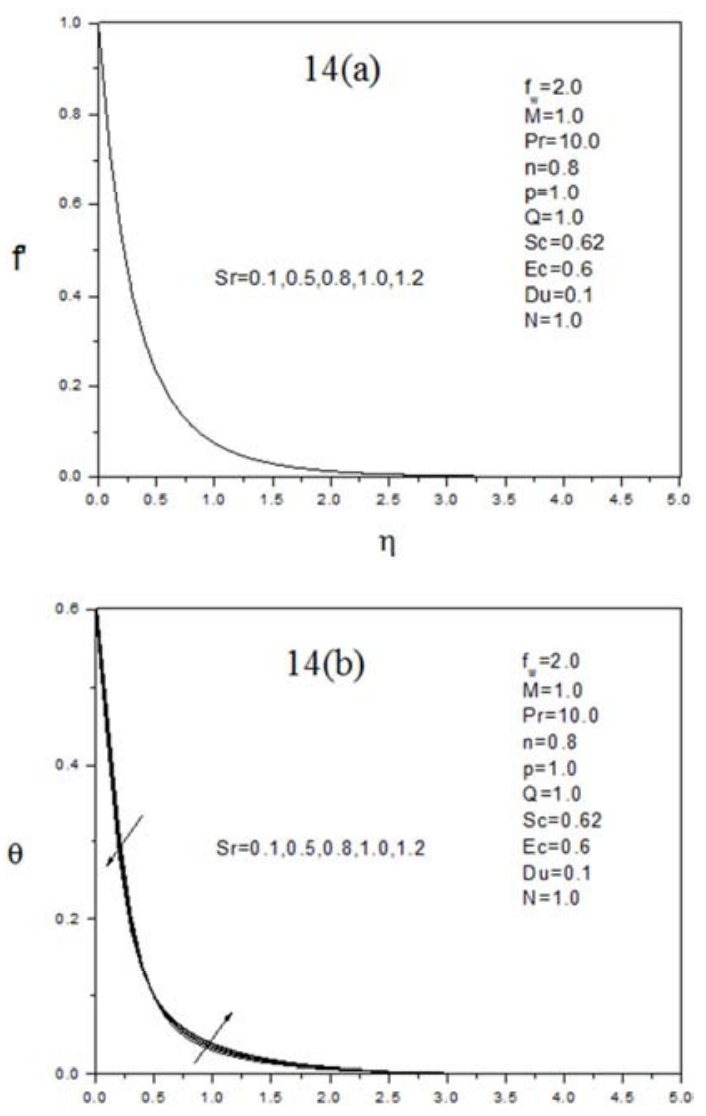

$\eta$

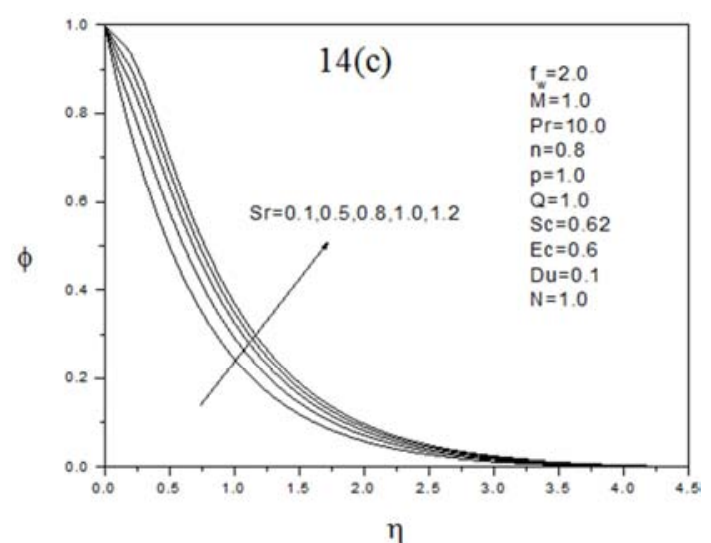

Fig. 14. (a), (b) and (c). Soret effect(Sr) on velocity, temperature and concentration profiles.

Due to suction some of the retarded fluid particles are taken out from the boundary layer and thus prevent the boundary layer separation. In Fig. 2(a), the velocity decreases with the increase of suction parameter. This can be explained by the fact that when the suction parameter $\left(f_{w}\right)$ increases, some matter is removed from the system and the velocity gets retarded most rapidly. Also the momentum boundary layer thickness decreases with the increase of the suction parameter $\left(f_{w}\right)$ and thus reduces the chance of the boundary layer to the transition to turbulence. From Fig. 2(b) and Fig. 2(c), there is similarity for temperature and concentration profiles that, the temperature and concentration of the fluid particle are low for a fluid of higher suction. The reduction in the velocity, temperature and concentration profiles also indicates that suction stabilizes the velocity, temperature and concentration boundary layer. So, suction can be used very effectively in controlling the flow field.

From Fig. 3(a), it is observed that there is no effect of Prandtl number on velocity profiles. This is because in the forced convection, the velocity is generally high in comparison with the effect of Prandtl number. The momentum boundary layer thickness remains fixed with the increase of the Prandtl number. So the velocity of the stretching sheet and the fluid particle remain same with the increase of Prandtl number Pr. From Fig. 3(b) the temperature decrease with the increase of the Prandtl number Pr, because of lower Prandtl number Pr, the heat transfer at much slower rate. On the other hand for higher Prandtl number Pr the heat transfer at much higher rate. The thermal boundary layer thickness decreases to a large extent with the increase of the Prandtl numberPr. However, for $\operatorname{Pr}=0.71,1.0$ wall temperature is very high compared to larger values. In Fig. 3(c) the concentration increases near the stretching sheet and then slowly decreases with the increase of Pr. It follows that Pr strongly influences the relative growth of the thermal and concentration boundary layer due to Dufour/Soret effects.

From Fig. 4(a), velocity decreases with the increase of magnetic number $(M)$. The induced magnetic field acts as a string on the flow field and retards the motion of the flow field. The consequence is that the velocity field decreases. Again from figure Fig. 4(b), with the increase of magnetic parameter $(M)$ the temperature increase and then finally start to decrease, so we get a cross flow near $\eta=0.3$. In Fig. 4(c), it is found that the magnetic parameter $M$ has significant increasing effect on concentration profiles due to Dufour $(D u)$ and Soret $(\mathrm{Sr})$ effects. It is observed from Fig. 5(a), Fig. 5(b), Fig.5(c) that the velocity, temperature and concentration profiles increase for the increase of the heat source parameter $(Q)$. This can be explained by the fact that with the increase of the heat source parameter $(Q)$, the thermal and concentration boundary layer thickness affected very rapidly. It indicates that heat source parameter $(Q)$ can be used very effectively to control the flow field. It is quite clear that concentration fields are responding due to Dufour $(\mathrm{Du})$ and Soret $(\mathrm{Sr})$ effects.

From Fig. 6(a), the velocity remains unchanged for the increase of the radiation parameter $(N)$. The momentum boundary layer thickness remains unaffected with the increase of the radiation parameter $(N)$. So the velocity of the stretching sheet and the fluid particle remain same with the increase of radiation parameter $(N)$. Thus there is no visible effect on velocity profiles. From Fig. 6(b) the temperature profiles decrease as the radiation parameter $(N)$ is increased. This can be explained by the fact that the thermal boundary layer thickness reduces very rapidly. It indicates that radiation effect can be used very effectively to control the temperature of the flow field. From Fig. 6(c) the concentration field firstly increases with the increase of radiation number $(N)$, then start to decrease. So we get a cross flow near $\eta=0.4$. With the increase of radiation parameter $(N)$, the temperature and concentration profiles stabilize quite slowly. So, we can say that $\operatorname{Dufour}(D u)$ and Soret $(\mathrm{Sr})$ effects are visible in the concentration profiles. 
Fig. 7(a), Fig. 7(b) and Fig. 7(c) exhibit the Schmidt number $(S c)$ on velocity, temperature and concentration profiles respectively. From the figures, it can be concluded that the velocity field remains unchanged for the increase of the Schmidt number $(S c)$. The momentum boundary layer thickness remains unaffected with the increase of the Schmidt number $(S c)$. It is seen from Fig.7(c) that the concentration profiles rapidly decrease with the Schmidt number $(S c)$. In case of temperature field, there is across flow near $\eta=0.5$. At first the temperature is higher near the stretching sheet and then slowly decreases. It indicates that for smaller values of Schmidt number $(S c)$ temperature stabilizes rather slowly. This happens due to $\operatorname{Dufour}(\mathrm{Du})$ and $\operatorname{Soret}(\mathrm{Sr})$ effects.

Fig. 8(a), Fig. 8(b) and Fig.8(c) show the Eckert number $(E c)$ on velocity, temperature and concentration profiles respectively. From the figures, we observe that the velocity remains unchanged for the increase of the Eckert number $(E c)$. The momentum boundary layer thickness remains unaffected with the increase of the Eckert number(Ec). In Fig. 8(b) and Fig. 8(c), it is observed that the temperature and concentration profiles rapidly increase with the Eckert number $(E c)$. It indicates that viscous dissipation effect can be used very effectively to control the temperature and concentration of the flow field. The concentration profiles are strongly affected due to $\operatorname{Dufour}(\mathrm{Du})$ and Soret $(\mathrm{Sr})$ effects.

Fig. 9(a), Fig. 10(a), Fig. 9(b), Fig. 10(b) and Fig. 9(c), Fig. 10(c) reveal the effects of velocity index $(p)$ for pseudoplastic fluids $(n<1)$ and dilatants fluids $(n>1)$ on velocity, temperature and concentration profiles respectively. So, we can conclude that velocity profiles decrease for both pseudoplastic $(n<1)$ and dilatants fluids $(n>1)$. But the temperature profiles in Fig. 9(b) and Fig. 10(b) show that the temperature profiles increase with the increase of velocity index for pseudo - plastic fluids $(n<1)$ and decrease with the increase of velocity index for dilatants fluids $(n>1)$. So, the pseudo-plastic fluids and dilatants fluids show completely opposite behavior for temperature profiles. In case of pseudoplastic and dilatants fluids, there is opposite visible effect on concentration field also. For dilatants fluids there is a very significant decreasing effect on concentration profiles and for pseudo-plastic fluids there is a rapid increase with the different values of velocity index $(p)$. For pseudo-plastic fluids $(n<1)$ the concentration profiles of the flow field are strongly affected due to $\operatorname{Dufour}(\mathrm{Du})$ and Soret $(\mathrm{Sr})$ effects. In case of dilatants fluids $(n>1)$, the Dufour $(D u)$ and Soret $(S r)$ effects reduce the growth of the boundary layer.

The effects of the velocity, temperature and concentration fields due to power-law fluid index $(n)$ for accelerated flow $(p=1.0)$ and decelerated flow $(p=-0.5)$ are shown in Fig. 11(a), Fig. 11(b), Fig. 11(c) and Fig. 12(a), Fig. 12(b), Fig. 12(c) respectively. From Fig. 11(a) and Fig. 12(a), with the increase of power-law fluid index $n$ the velocity increase then start to decrease, so we get a cross flow near $\eta=1.0$. In Fig. 11(b) with the increase of power-law fluid index $n$ the temperature increase then start to decrease, so we get a cross flow near $\eta=0.5$. The temperature profiles increase with the increase of the power-law fluid index $(n)$ for decelerated fluid in Fig. 12(b). Here, Fig. 11(c) and Fig. 12(c), there is opposite effect on concentration profile for accelerated flow $(p=1.0)$ and decelerated flow $(p=-0.5)$. The concentration profiles sharply decrease for accelerated flow $(p=1.0)$ and increase for decelerated flow $(p=-0.5)$ with the increase of powerlaw fluid index $(n)$. So, Dufour $(D u)$ and Soret $(S r)$ effects reduce the growth of the momentum boundary layer. But the highly effecting behavior is shown in temperature profiles due to $\operatorname{Dufour}(\mathrm{Du})$ and $\operatorname{Soret}(\mathrm{Sr})$ effects.

Fig. 13(a), Fig. 13(b) and Fig. 13(c) show the effects of Dufour number $(D u)$ on velocity, temperature and concentration profiles respectively. From the figures, we observe that the velocity remains unchanged for the increase of the Dufour number $(D u)$. The momentum boundary layer thickness remains unaffected with the increase of the Dufour number $(D u)$. In Fig. 13(b) and Fig. 13(c) we see that the temperature and concentration profiles rapidly increase with the Dufour number $(D u)$. The heat and mass transfer rate are strongly influenced with the increasing Dufour number. So, the temperature and concentration of the flow field can be controlled with Dufour number $(D u)$. This explains by the fact that Dufour number $(\mathrm{Du})$ shows excellent mutual interaction between temperature and concentration of the flow field.

From Fig. 14(a), Fig. 14(b) and Fig. 14(c), the effects of Soret number $(\mathrm{Sr})$ on velocity, temperature and concentration profiles are shown respectively. From this figures, it is visualized that the velocity remains unchanged for the increase of the Soret number $(\mathrm{Sr})$. The momentum boundary layer thickness remains unaffected with the increase of the Soret number(Sr). In Fig. 14(b) the temperature profiles first start to decrease and then began to increase. We indicate a cross flow near $\eta=0.5$. From Fig. 14(c) indicates the remarkable effect on concentration profiles. There is a very sharp rise on concentration profiles near the stretching sheet that indicates that Soret number $(\mathrm{Sr})$ controls concentration boundary layer. From above investigations, we can say that Soret number $(\mathrm{Sr})$ has powerful mutual interacting effect on temperature profiles of the flow field.

\section{Skin Friction Coefficient $\left(\boldsymbol{C}_{\boldsymbol{f}}\right)$, Local Nusselt Number $\left(N u_{x}\right)$ and Local Sherwood Number $(S h)$}

$$
\begin{array}{r}
\text { The wall shear stress, } \tau_{w}=\left.\mathrm{K}\left(\left|\frac{\partial \mathrm{u}}{\partial \mathrm{y}}\right|^{\mathrm{n}-1} \frac{\partial \mathrm{u}}{\partial \mathrm{y}}\right)\right|_{\mathrm{y}=0} \\
=\rho u_{w}{ }^{2} R e_{x}^{-\frac{1}{(n+1)}}\left|f^{\prime \prime}(0)\right|^{n-1} f^{\prime \prime}(0) .
\end{array}
$$

So, Skin friction coefficient, $\mathrm{C}_{\mathrm{f}}=\frac{\tau_{w}}{\frac{1}{2} \rho u_{w}^{2}}$. Or, $R e_{x}^{\frac{1}{(n+1)}} \mathrm{C}_{\mathrm{f}}=$ $2\left|f^{\prime \prime}(0)\right|^{n-1} f^{\prime \prime}(0)$. The local Nusselt number, $N u_{x}=\frac{h x}{k}=$ $\frac{R e_{x}^{1 /(n+1)}}{\theta(0)}$ or, $R e_{x}^{\frac{-1}{(n+1)}} N u_{x}=\frac{1}{\theta(0)}$ and local Sherwood number, $S h=\frac{x M_{w}}{D_{m}\left(C-C_{\infty}\right)}$ or, $S h R e_{x}^{\frac{-1}{(n+1)}}=-\phi^{\prime}(0)$. Here, skin friction coefficient $\left(C_{f}\right)$, local Nusselt number $\left(N u_{x}\right)$ and local Sherwood number (Sh) are proportional to $2\left|f^{\prime \prime}(0)\right|^{n-1} f^{\prime \prime}(0), 1 / \theta(0)$ and $-\phi^{\prime}(0)$ respectively. The effects of Dufour and Soret number on skin friction 
coefficient $\left(C_{f}\right)$, local Nusselt number $\left(N u_{x}\right)$ and local Sherwood number(Sh) are shown in tabular and graphical form.
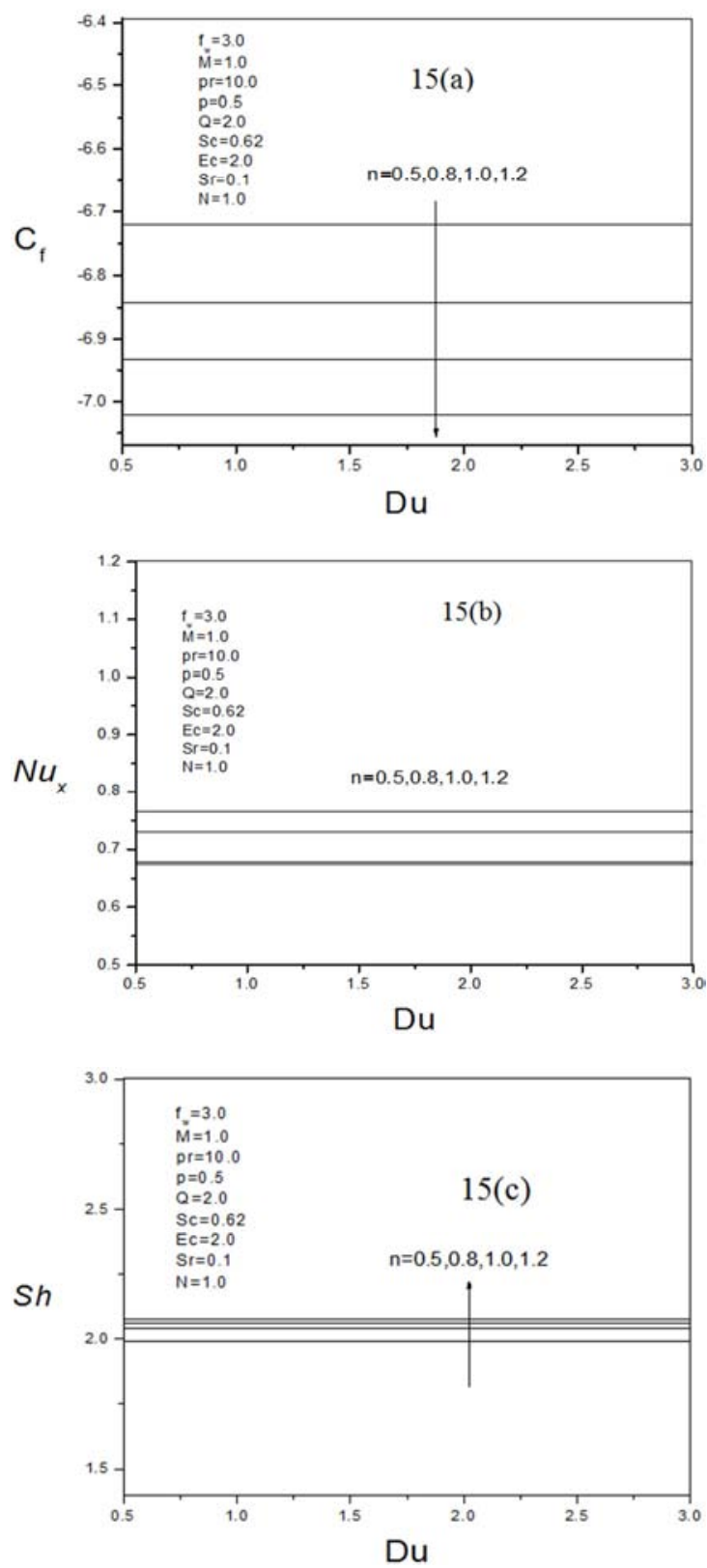

Fig. 15. (a), (b and (c). Variation of skin friction coefficient $\left(C_{f}\right)$, local Nusselt number $\left(N u_{x}\right)$ and local Sherwood number $(\mathrm{Sh})$ as a function of (Du).

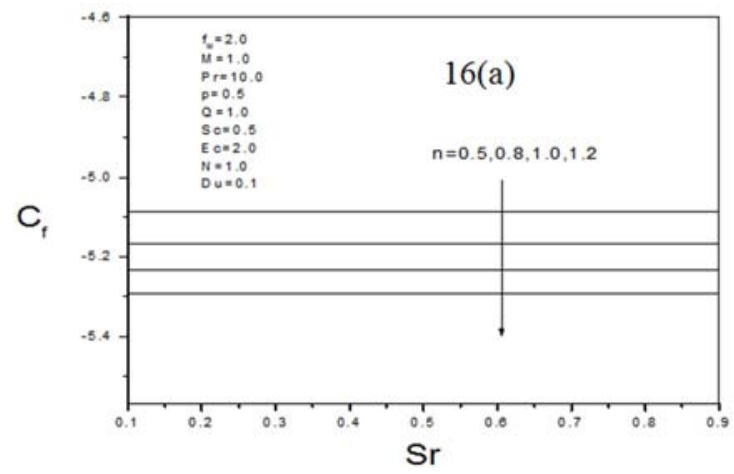

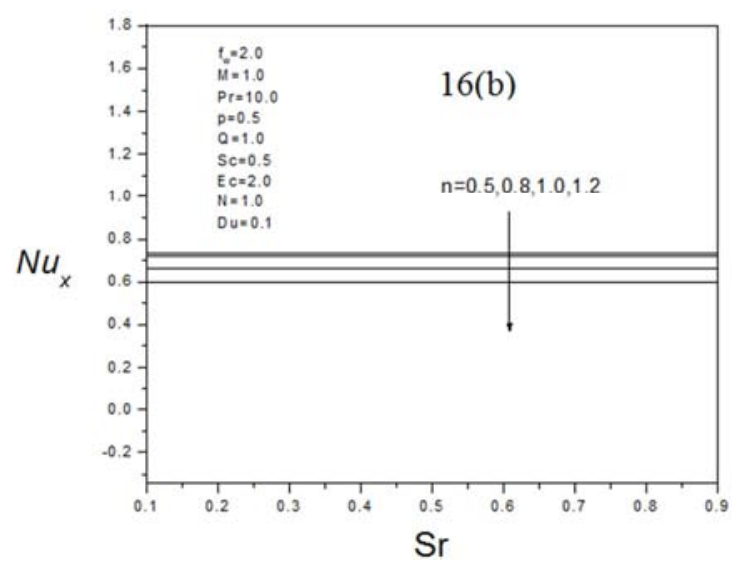

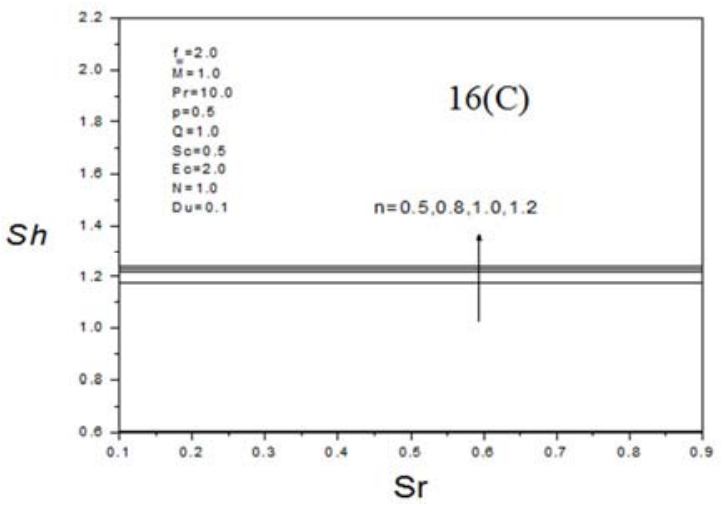

Fig. 16. (a), (b) and (c). Variation of skin friction coefficient $\left(C_{f}\right)$, local Nusselt number $\left(N u_{x}\right)$ and local Sherwood number $(\mathrm{Sh})$ as a function of $(\mathrm{Sr})$.
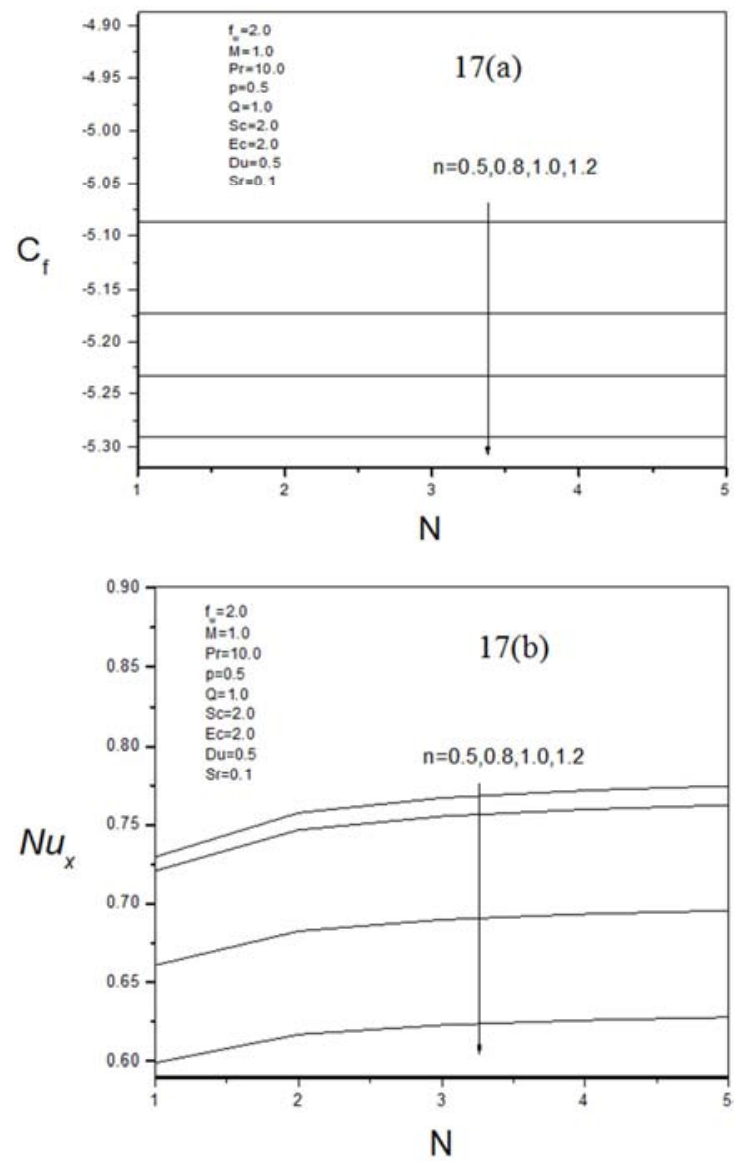


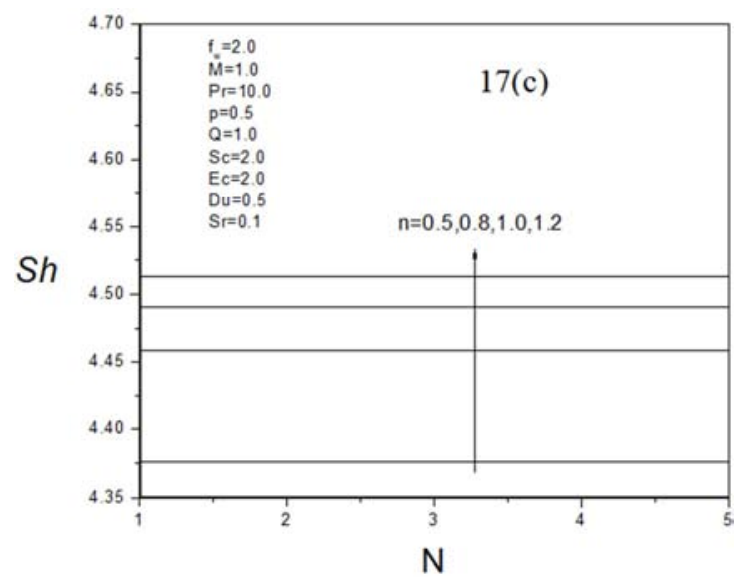

Fig. 17. (a), (b) and (c). Variation of skin friction coefficient $\left(C_{f}\right)$, local Nusselt number $\left(N u_{x}\right)$ and local Sherwood number $(\mathrm{Sh})$ as a function of $(N)$.

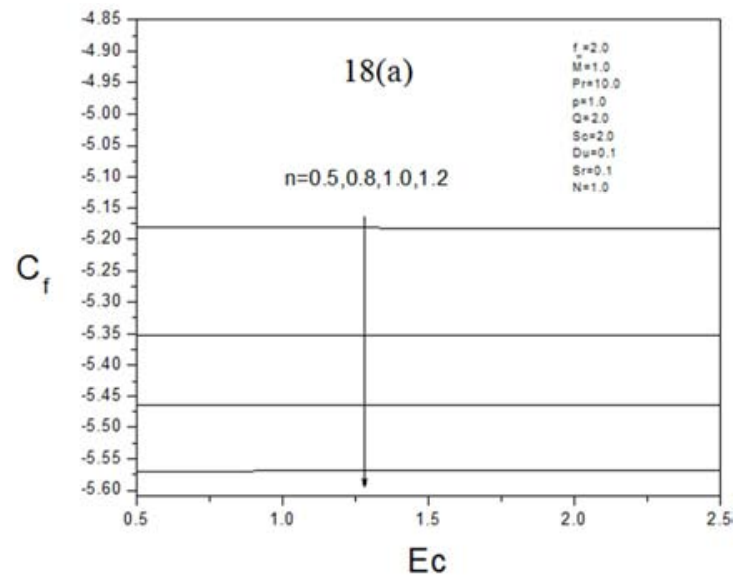

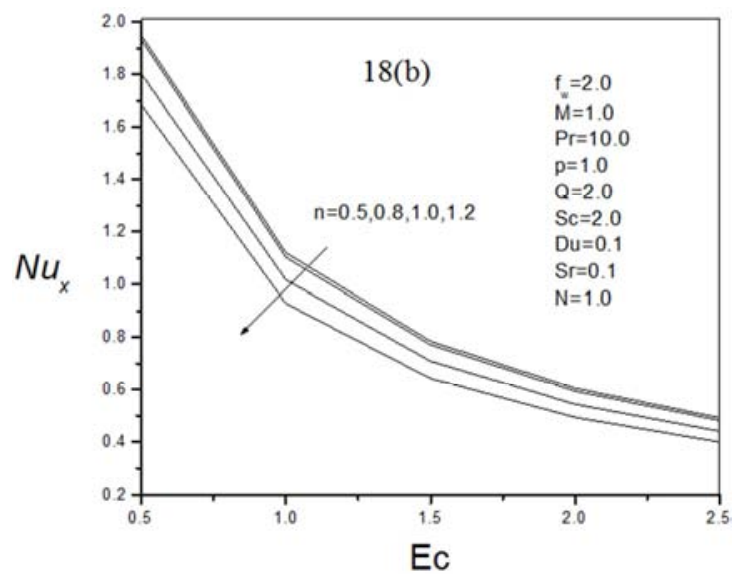

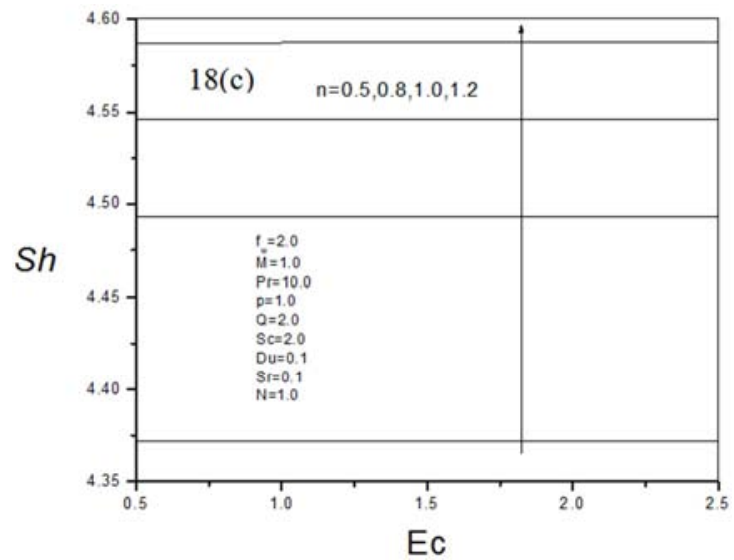

Fig. 18. (a), (b) and (c). Variation of skin friction coefficient $\left(C_{f}\right)$, local Nusselt number $\left(N u_{x}\right)$ and local Sherwood number $(\mathrm{Sh})$ as a function of $(E C)$.

Table 1. Skin friction coefficient $\left(C_{f}\right)$, local Nusselt number $\left(N u_{x}\right)$ and local Sherwood number $(S h)$ for different values of Dufour number $(D u)$

\begin{tabular}{|c|c|c|c|c|c|c|c|c|c|}
\hline$n$ & $D u$ & $\operatorname{Re}_{x}^{\frac{1}{(n+1)}} \mathrm{C}_{\mathrm{f}}$ & $R e_{x}^{\frac{-1}{(n+1)}} N u_{x}$ & $\operatorname{ShR} e_{x}^{\frac{-1}{(n+1)}}$ & $n$ & $D u$ & $\operatorname{Re}_{x}^{\frac{1}{(n+1)}} \mathrm{C}_{\mathrm{f}}$ & $R e_{x}^{\frac{-1}{(n+1)}} N u_{x}$ & $\operatorname{ShR} e_{x}^{\frac{-1}{(n+1)}}$ \\
\hline \multirow{6}{*}{0.5} & 0.5 & -6.7200163 & 0.6751915 & 1.9922553 & \multirow{6}{*}{1.0} & 0.5 & -6.9335125 & 0.7303696 & 2.0599073 \\
\hline & 1.0 & -6.7200163 & 0.6751915 & 1.9922553 & & 1.0 & -6.9335125 & 0.7303696 & 2.0599073 \\
\hline & 1.5 & -6.7200163 & 0.6751915 & 1.9922553 & & 1.5 & -6.9335125 & 0.7303696 & 2.0599073 \\
\hline & 2.0 & -6.7200163 & 0.6751915 & 1.9922553 & & 2.0 & -6.9335125 & 0.7303696 & 2.0599073 \\
\hline & 2.5 & -6.7200163 & 0.6751915 & 1.9922553 & & 2.5 & -6.9335125 & 0.7303696 & 2.0599073 \\
\hline & 3.0 & -6.7200163 & 0.6751915 & 1.9922553 & & 3.0 & -6.9335125 & 0.7303696 & 2.0599073 \\
\hline \multirow{6}{*}{0.8} & 0.5 & -6.8427373 & 0.7649702 & 2.0402050 & \multirow{6}{*}{1.2} & 0.5 & -7.0202541 & 0.6786496 & 2.0750089 \\
\hline & 1.0 & -6.8427373 & 0.7649702 & 2.0402050 & & 1.0 & -7.0202541 & 0.6786496 & 2.0750089 \\
\hline & 1.5 & -6.8427373 & 0.7649702 & 2.0402050 & & 1.5 & -7.0202541 & 0.6786496 & 2.0750089 \\
\hline & 2.0 & -6.8427373 & 0.7649702 & 2.0402050 & & 2.0 & -7.0202541 & 0.6786496 & 2.0750089 \\
\hline & 2.5 & -6.8427373 & 0.7649702 & 2.0402050 & & 2.5 & -7.0202541 & 0.6786496 & 2.0750089 \\
\hline & 3.0 & -6.8427373 & 0.7649702 & 2.0402050 & & 3.0 & -7.0202541 & 0.6786496 & 2.0750089 \\
\hline
\end{tabular}

Table 2. Skin friction coefficient $\left(C_{f}\right)$, local Nusselt number $\left(N u_{x}\right)$ and local Sherwood number $(S h)$ for different values of Soret number $(S r)$

\begin{tabular}{|c|c|c|c|c|c|c|c|c|c|}
\hline$n$ & $S r$ & $R e_{x}^{\frac{1}{(n+1)}} C_{f}$ & $R e_{x}^{\frac{-1}{(n+1)}} N u_{x}$ & $\operatorname{ShR} e_{x}^{\frac{-1}{(n+1)}}$ & $n$ & $S r$ & $R e_{x}^{\frac{1}{(n+1)}} C_{f}$ & $R e_{x}^{\frac{-1}{(n+1)}} N u_{x}$ & $\operatorname{ShRe}_{x}^{\frac{-1}{(n+1)}}$ \\
\hline \multirow{5}{*}{0.5} & 0.1 & -5.0870808 & 0.7322962 & 1.1767294 & \multirow{5}{*}{1.0} & 0.1 & -5.2332202 & 0.6608245 & 1.2312019 \\
\hline & 0.3 & -5.0870808 & 0.7322962 & 1.1767294 & & 0.3 & -5.2332202 & 0.6608245 & 1.2312019 \\
\hline & 0.5 & -5.0870808 & 0.7322962 & 1.1767294 & & 0.5 & -5.2332202 & 0.6608245 & 1.2312019 \\
\hline & 0.7 & -5.0870808 & 0.7322962 & 1.1767294 & & 0.7 & -5.2332202 & 0.6608245 & 1.2312019 \\
\hline & 0.9 & -5.0870808 & 0.7322962 & 1.1767294 & & 0.9 & -5.2332202 & 0.6608245 & 1.2312019 \\
\hline \multirow{5}{*}{0.8} & 0.1 & -5.1696151 & 0.7219353 & 1.2165534 & \multirow{5}{*}{1.2} & 0.1 & -5.2935088 & 0.5981121 & 1.2420021 \\
\hline & 0.3 & -5.1696151 & 0.7219353 & 1.2165534 & & 0.3 & -5.2935088 & 0.5981121 & 1.2420021 \\
\hline & 0.5 & -5.1696151 & 0.7219353 & 1.2165534 & & 0.5 & -5.2935088 & 0.5981121 & 1.2420021 \\
\hline & 0.7 & -5.1696151 & 0.7219353 & 1.2165534 & & 0.7 & -5.2935088 & 0.5981121 & 1.2420021 \\
\hline & 0.9 & -5.1696151 & 0.7219353 & 1.2165534 & & 0.9 & -5.2935088 & 0.5981121 & 1.2420021 \\
\hline
\end{tabular}


Table 3. Skin friction coefficient $\left(C_{f}\right)$, local Nusselt number $\left(N u_{x}\right)$ and local Sherwood number $(S h)$ for different values of Radiation parameter $(N)$.

\begin{tabular}{|c|c|c|c|c|c|c|c|c|c|}
\hline$n$ & $N$ & $R e_{x}^{\frac{1}{(n+1)}} C_{\mathrm{f}}$ & $R e_{x}^{\frac{-1}{(n+1)}} N u_{x}$ & $\operatorname{ShR}_{x}^{\frac{-1}{(n+1)}}$ & $n$ & $N$ & $R e_{x}^{\frac{1}{(n+1)}} \mathrm{C}_{\mathrm{f}}$ & $R e_{x}^{\frac{-1}{(n+1)}} N u_{x}$ & $\operatorname{Sh} R e_{x}^{\frac{-1}{(n+1)}}$ \\
\hline \multirow{5}{*}{0.5} & 1.0 & -5.0871219 & 0.7296903 & 4.3762276 & \multirow{5}{*}{1.0} & 1.0 & -5.2331558 & 0.6608649 & 4.4904008 \\
\hline & 2.0 & -5.0871215 & 0.7576605 & 4.3762277 & & 2.0 & -5.2331557 & 0.6825725 & 4.4904008 \\
\hline & 3.0 & -5.0871213 & 0.7671051 & 4.3762277 & & 3.0 & -5.2331556 & 0.6898649 & 4.4904008 \\
\hline & 4.0 & -5.0871212 & 0.7718475 & 4.3762277 & & 4.0 & -5.2331556 & 0.6935207 & 4.4904008 \\
\hline & 5.0 & -5.0871212 & 0.7746989 & 4.3762277 & & 5.0 & -5.2331556 & 0.6957170 & 4.4904008 \\
\hline \multirow{5}{*}{0.8} & 1.0 & -5.1727418 & 0.7209275 & 4.4589425 & \multirow{5}{*}{1.2} & 1.0 & -5.2911746 & 0.5987634 & 4.5130057 \\
\hline & 2.0 & -5.1727417 & 0.7467661 & 4.4589425 & & 2.0 & -5.2911744 & 0.6167403 & 4.5130057 \\
\hline & 3.0 & -5.1727417 & 0.7554826 & 4.4589425 & & 3.0 & -5.2911744 & 0.6227563 & 4.5130057 \\
\hline & 4.0 & -5.1727417 & 0.7598590 & 4.4589425 & & 4.0 & -5.2911744 & 0.6257674 & 4.5130057 \\
\hline & 5.0 & -5.1727417 & 0.7624903 & 4.4589425 & & 5.0 & -5.2911744 & 0.6275748 & 4.5130057 \\
\hline
\end{tabular}

Table 4. Skin friction coefficient $\left(C_{f}\right)$, local Nusselt number $\left(N u_{x}\right)$ and local Sherwood number $(S h)$ for different values of Eckert number $(E c)$.

\begin{tabular}{|c|c|c|c|c|c|c|c|c|c|}
\hline$n$ & $\boldsymbol{E} \boldsymbol{C}$ & $R e_{x}^{\frac{1}{(n+1)}} \mathrm{C}_{\mathrm{f}}$ & $R e_{x}^{\frac{-1}{(n+1)}} N u_{x}$ & $\operatorname{ShR}_{x}^{\frac{-1}{(n+1)}}$ & $\boldsymbol{n}$ & $\boldsymbol{E} \boldsymbol{c}$ & $R e_{x}^{\frac{1}{(n+1)}} C_{f}$ & $R e_{x}^{\frac{-1}{(n+1)}} N u_{x}$ & $\operatorname{ShR} e_{x}^{\frac{-1}{(n+1)}}$ \\
\hline \multirow{5}{*}{0.5} & 0.5 & -5.1822602 & 1.9513356 & $4.3718910^{x}$ & \multirow{5}{*}{1.0} & 0.5 & $\begin{array}{c}x \\
-5.4640273\end{array}$ & 1.8037369 & 4.5461965 \\
\hline & 1.0 & -5.1822646 & 1.1197732 & 4.3718905 & & 1.0 & -5.4640273 & 1.0176644 & 4.5461965 \\
\hline & 1.5 & -5.1822719 & 0.7852214 & 4.3718896 & & 1.5 & -5.4640274 & 0.7087775 & 4.5461965 \\
\hline & 2.0 & -5.1822819 & 0.6046329 & 4.3718884 & & 2.0 & -5.4640274 & 0.5437390 & 4.5461965 \\
\hline & 2.5 & -5.1822946 & 0.4916149 & 4.3718868 & & 2.5 & -5.4640275 & 0.4410425 & 4.5461965 \\
\hline \multirow{4}{*}{0.8} & 0.5 & -5.3525880 & 1.9342283 & 4.4928784 & \multirow{4}{*}{1.2} & 0.5 & -5.5713896 & 1.6877775 & 4.5871800 \\
\hline & 1.0 & -5.3525880 & 1.1038137 & 4.4928784 & & 1.0 & -5.5688401 & 0.9285053 & 4.5873033 \\
\hline & 2.0 & -5.3525882 & 0.5938785 & 4.4928784 & & 2.0 & -5.5687937 & 0.4941274 & 4.5873049 \\
\hline & 2.5 & -5.3525883 & 0.4824406 & 4.4928784 & & 2.5 & -5.5688383 & 0.3992198 & 4.5873033 \\
\hline
\end{tabular}

\section{Conclusion}

Under the Dufour and Soret effects, Suction has significant effects on the flow field and can be used to control the boundary layer growth. In case of forced convection, the velocity is large. So, the Prandtl number (Pr) has no effective dominance over velocity field. But it has significant effect on the temperature and concentration fields. With the increase of magnetic parameter $(M)$ the temperature increase then start to decrease, so we get a cross flow and an increasing behavior are shown in concentration profiles. The temperature and concentration profiles increase with the heat source parameter $(Q)$ is increased. The concentration profiles rapidly decrease with the Schmidt number $(S c)$ and for smaller values of Schmidt number $(S c)$ temperature stabilizes rather slowly. The temperature and concentration profile rapidly increase with the Eckert number $(E c)$. In case of velocity index $(p)$, the pseudo-plastic and dilatants fluids show completely opposite behavior for temperature and concentration profile. Due to power-law fluid index $(n)$ for accelerated $(p=1.0)$ and decelerated $(p=-0.5)$ flows, there is an opposite behavior in the concentration field. Dufour $(\mathrm{Du})$ and Soret $(\mathrm{Sr})$ numbers show excellent mutual interaction between temperature and concentration of the flow field.

\section{References}

[1] Elbashbeshy, E. M. A., "Heat Transfer over a Stretching Surface with Variable Surface Heat Flux", J. Phys. D: Appl. Phys. 31(1998), 1951-1954.

[2] Vajravelu, K. and A. Hadjinicolaou, "Convective Heat Transfer in an Electrically Conducting Fluid at a stretching surface with uniform free stream”, Int. J. Eng. Sci. 35(1997), 12-13, 1237-1244.

[3] Howell, T. G., D. R. Jeng, and K. J. De Witt, "Momentum and heat transfer on a continuous moving surface in a power law fluid", Int. J. of Heat and Mass Transfer, 40 (1997), 18531861.

[4] Rahman, M. A., M. A. Samad, M. M. Rahman and M. Muhebujjaman, "Numerical Study of MHD Forced Convective Flow of a Micropolar Fluid Past a Non-Linear Stretching Sheet with a Variable Viscosity", Dhaka Univ. J. Sci. 57 (2008), 243-248.

[5] Raptis, A., "Flow of a micropolar fluid past a continuously moving plate by the presence of radiation", Int. J. Heat Mass Transfer, 41 (1998), 2865-2866.

[6] Andersson, H. I., K. H. Bech, and B. S. Dandapat, "Magnetohydrodynamic flow of a power-law fluid over a stretching sheet”, Int. J. Non-Linear Mech. 27 (1992), 926936.

[7] Mahmoud, M. A. A. and M. A. E. Mahmoud, "Analytical solutions of hydromagnetic Boundary-Layer flow of a nonNewtonian power-law fluid past a continuously moving surface", Acta Mechanica, 181 (2006), 83-89.

[8] Dandapaat, B. S. and A. S. Gupta, "Flow and heat transfer in a viscoelastic fluid over a stretching sheet", Int. J. Non-Linear Mech. 40 (2005), 215-219.

[9] Datti, P. S., K. V. Prasad, M. S. Abel, and A. Joshi, "MHD viscoelastic fluid flow over a non-isothermal stretching sheet", Int. J. Eng. Sci. 42 (2005), 935-946.

[10] Cess, R. D., "The effect of Radiation upon the forced convection heat transfer", Appl. Sci. Res. 10 (1966), 12691277.

[11] Pop, S. R., T. Grosan and I. Pop, "Radiation Effect on the Flow Near the Stagnation Point of a Stretching Sheet", Technische Mechanik, 25 (2004), 100-106. 
[12] Damesh, R. A., H. M. Duwairi and M. Al-Odat, "Similarity analysis of magnetic field and thermal radiation effects on forced convection flow", Turk J. Eng Env. 30 (2006), 83-89.

[13] Cortell, R., "Effects of viscous dissipation and radiation on the thermal boundary layer over a nonlinearly stretching sheet", Physics Letters, 372 (2008), 631-636.

[14] Chen, C. H., "Effects of magnetic field and suction/injection on convective heat transfer on non-Newtonian power-law fluids past a power-law stretched sheet with surface heat flux", Int. J. Thermal Sci. 47 (2008), 954-961.

[15] O. D. Makinde, K. Zimba, O. Anwar Beg, "Numerical Study of Chemically-Reacting Hydromagnetic Boundary Layer Flow with Soret/Dufour Effects and a Convective Surface Boundary Condition", Int. J. of Thermal \& Environmental Engineering, 4 (1) (2012), 89-98.

[16] M. J. Subhakar, K. Gangadhar, N. Bhaskar Reddy, "Soret and Dufour effects on MHD convective flow of heat and mass transfer over a moving non-isothermal vertical plate with heat generation/absorption", Advances in Applied Science Research, 3 (5) (2012), 3165-3184.

[17] Alam M. S., M. M. Rahman, M. A. Samad, "Dufour and Soret effects on unsteady MHD Free convection and Mass Transfer flow past a vertical porous plate in a porous medium", Nonlinear Analysis: Modelling and Control, 11 (3) (2006): 217-226.
[18] Mahdy, A., "Soret and Dufour effect on double diffusion mixed convection from a vertical surface in a porous medium saturated with a non-Newtonian fluid", J. of Non-Newtonian Fluid Mech., 165 (2010): 568-575.

[19] Tai Bo-Chen and Char Ming-I, "Soret and Dufour effects on free convection flow of non-Newtonian fluids along a vertical plate embedded in a porous medium with thermal radiation", Int. Commun. Heat and Mass Transfer, 37 (2010): 480-483.

[20] Abreu C. R. A., Alfradique, M. F. and Silva, A. T., "Boundary layer flows with Dufour and Soret effects: I: Forced and natural convection", Chemical Engineering Science, 61(2006): 4282-4289.

[21] Moorthy M. B. K., Kannan T. and Senthil Vaido, "Soret and Dufour Effects on Natural Convection Heat and Mass Transfer Flow past a Horizontal Surface in a Porous Medium with Variable Viscosity", WSEASTRANSACTIONS on HEAT and MASS TRANSFER, 8(3) (2013):121-130.

[22] Omowaye A. J., Fagbade A. I and Ajayi A. O., "Dufour and soret effects on steady MHD convective flow of a fluid in a porous medium with temperature dependent viscosity: Homotopy analysis approach", Journal of the Nigerian Mathematical Society, 34 (2015): 343-360. 